\title{
Magnetic fields in elliptical galaxies: Using the Laing-Garrington effect in radio galaxies and polarized emission from background radio sources
}

\author{
Hilay Shah ${ }^{1 \star}$ and Amit $\operatorname{Seta}^{2} \dagger$ \\ ${ }^{1}$ Department of Physics, Indian Institute of Technology Roorkee, 247667, India \\ ${ }^{2}$ Research School of Astronomy and Astrophysics, Australian National University, Canberra, ACT 2611, Australia
}

Accepted XXX. Received YYY; in original form ZZZ

\begin{abstract}
Magnetic fields in elliptical galaxies are poorly constrained due to a lack of significant synchrotron emission from them. This paper explores properties of magnetic fields in ellipticals via two methods. First, we exploit the Laing-Garrington effect (asymmetry in the observed polarization fraction between radio galaxy jets) for 57 galaxies with redshifts up to 0.5 . We use the differences in polarization fraction and rotation measure between the jet and counterjet to estimate the small- and large-scale magnetic fields in and around ellipticals (including their circumgalactic medium). We find that the small-scale field (at scales smaller than the driving scale of turbulence, approximately $300 \mathrm{pc}$ ) lies in the range $0.1-2.75 \mu \mathrm{G}$. The large-scale field (at scales of $100 \mathrm{kpc}$ ) is an order of magnitude smaller than the small-scale field. In the second method, we cross-match the Faraday rotation measures (RM) of a few hundred (out of 3098) extragalactic radio sources with galaxy catalogs to explore the effect of the number and morphology of intervening galaxies on the observed RM distribution. We use both Gaussian and non-Gaussian functions to describe the RM distribution and derive its statistical properties. Finally, using the difference in the observed polarization fraction between the intervening spirals and ellipticals, we estimate the small-scale magnetic fields at the center of ellipticals to be $\sim 6 \mu \mathrm{G}$. Both methods with different observations and analysis techniques give magnetic field strengths consistent with previous studies $(\leq 10 \mu \mathrm{G})$, and the results can be used to constrain dynamo theories and galaxy evolution simulations.
\end{abstract}

Key words: magnetic fields - dynamo - galaxies: magnetic fields - galaxies: elliptical and lenticular, cD - galaxies: high-redshift - techniques: polarimetric

\section{INTRODUCTION}

Magnetic fields are ubiquitous in the universe and are a key ingredient in cosmic evolution and structure formation. They can permeate smaller astrophysical objects $\left(10^{-10}-10^{-8} \mathrm{pc}\right)$ such as planets and stars and also objects on much larger-scales $\left(10^{2}-10^{4} \mathrm{pc}\right)$ such as the interstellar and intergalactic medium (ISM and IGM). Their role in galaxy formation and evolution is not completely known yet, but recent cosmological simulations suggest that they are an important component of galaxy evolution (van de Voort et al. 2021). However, their influence on the physical processes in galaxies such as star formation (Birnboim et al. 2015; Krumholz \& Federrath 2019), heating of the ISM (Raymond 1992), launching of galactic winds (Evirgen et al. 2017; Hopkins et al. 2018; van de Voort et al. 2021), and propagation of cosmic rays (Cesarsky 1980; Shukurov et al. 2017; Seta et al. 2018) is anything but negligible.

Understanding the impact of magnetic fields on such processes requires details of their origin, strength, spatial scales, and evolution over galactic and cosmic time scales. Dynamo theory (Larmor 1919; Herzenberg 1958; Brandenburg \& Subramanian 2005; Federrath 2016; Rincon 2019), a process by which the kinetic energy of the medium is converted to magnetic energy, is widely accepted as

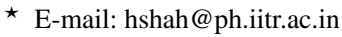

$\dagger$ E-mail: amit.seta@anu.edu.au
}

the magnetic field amplification mechanism in astrophysical objects (Beck et al. 1996; Brandenburg \& Subramanian 2005). Most astrophysical systems are turbulent, and without a self-sustaining dynamo process, turbulent magnetic diffusion quickly destroys the seed magnetic fields in a fraction of the galactic lifetime (Shukurov \& Sokoloff 2007). For example, in spiral galaxies, the decay time scale of the magnetic fields due to the turbulent diffusion is of the order of $10^{8} \mathrm{yr}$, whereas the typical lifetime of a spiral galaxy is around $10^{10} \mathrm{yr}$ (see Sec. 1.5 in Seta 2019). So, the observed astrophysical magnetic fields are amplified and maintained by a dynamo mechanism.

Most previous studies of galactic magnetic fields deal with spiral galaxies (Beck 2015). The magnetic fields in spiral galaxies $(B)$ are divided into large-scale or mean fields $(\bar{B}$, ordered over $\mathrm{kpc}$ scales) and small-scale or fluctuating fields ( $b$, correlation length less than the driving scale of turbulence, $\lesssim 100 \mathrm{pc}$ ). In the presence of magnetic fields, cosmic ray electrons, generated by star-formation, supernovae events, active galactic nuclei (AGN), $\gamma$-ray bursts, emit synchrotron radiation which is intrinsically linearly polarized (Duric 1988; Klein \& Fletcher 2015). The synchrotron intensity traces the total magnetic field perpendicular to the line of sight. The polarization angle $\psi_{0}$ of a linearly polarized synchrotron radiation wave emitted by a radio source is rotated in the magneto-ionic medium along the line of sight, at a wavelength $\lambda$ (see Sec. 3.3 in Klein \& 
Fletcher 2015) such that:

$$
\psi_{\mathrm{obs}}=\psi_{0}+\mathrm{RM} \lambda^{2},
$$

where $\psi_{\mathrm{obs}}$ is the observed polarization angle, and RM is the Faraday rotation measure. RM in the observer's frame is related to the properties of the magneto-ionic medium by the equation

$$
\mathrm{RM}=\frac{0.81}{(1+z)^{2}} \int_{0}^{L} \frac{n_{e}(z, l)}{\mathrm{cm}^{-3}} \frac{\mathbf{B}(z, l)}{\mu \mathrm{G}} \cdot \frac{\mathrm{d} \mathbf{l}}{\mathrm{pc}} \mathrm{rad} / \mathrm{m}^{2},
$$

where $z$ is the redshift of the source, $n_{\mathrm{e}}\left(\mathrm{cm}^{-3}\right)$ is the thermal electron density, $\mathbf{B}(\mu \mathrm{G})$ is the magnetic field, and $\mathrm{d} l$ is an infinitesimal element along the line of sight (LOS) with $L$ being the total path length. The observed Faraday rotation measure (RM) provides information about the magnetic field component parallel to the line of sight, where the RM values directly probe the ordered field, which is the large-scale field component for our study due to substantially large path lengths.

When random fluctuations of magnetic fields (small-scale magnetic fields) are present in a medium, the polarization signal can be reduced due to various depolarization mechanisms, (Sokoloff et al. 1998) and it also leads to random fluctuations in RM. Thus, information about the small-scale fields can be obtained from the depolarization measurements and the fluctuations in RM (given sufficient spatial resolution). In the Milky Way and nearby spiral galaxies, depending on the galaxy and the region within it, the total field strength lies in the range $5-25 \mu \mathrm{G}$ with the small-scale field stronger than the large-scale by a factor of $1-5$ (Fletcher 2010; Beck 2015).

Motivated by observational results, the dynamo theory is also conventionally divided into small- and large-scale dynamos. The smallscale dynamo only requires turbulence and a very modest value of the magnetic Reynolds number $\left(\sim 10^{2}-10^{3}\right)$ to generate small-scale fields (Kazantsev 1968; Schekochihin et al. 2004; Brandenburg \& Subramanian 2005; Seta et al. 2020). The large-scale dynamo requires turbulence and large-scale scale properties of galaxies such as differential rotation, shear, and density stratification to generate fields over kpc scales (Ruzmaikin et al. 1988; Blackman 1998; Brandenburg \& Subramanian 2005; Shukurov \& Sokoloff 2007).

Besides the small-scale dynamo, small-scale random magnetic fields in spiral galaxies can also originate from the following two mechanisms: tangling of the large-scale field due to turbulence (Sec. 4.1 in Seta \& Federrath 2020) and amplification of small-scale magnetic fields due to compressive motions (Federrath et al. 2011, 2014). In spiral galaxies, turbulence is driven at a range of scales by a variety of phenomena, like from molecular cloud scales $(\sim 1-10 \mathrm{pc})$ by stellar winds (Mac Low \& Klessen 2004) to galaxy scales ( $\mathrm{kpcs}$ ) by gravitational instabilities (Krumholz et al. 2018). However, the most energetic events in the ISM of spiral galaxies are supernova explosions, which drive turbulence at scales of around $100 \mathrm{pc}$ (roughly the order of the size of a typical supernova remnant). The characteristic scale of small-scale magnetic fields is around $50 \mathrm{pc}$ (Gaensler et al. 2005; Fletcher et al. 2011; Beck 2015). The present-day telescope resolution in spiral galaxies is hundreds of pc in radio bands $(3-21 \mathrm{~cm})$ (Beck 2007; Tabatabaei et al. 2008; Kierdorf et al. 2020) with the Effelsberg telescope and Very Large Array (VLA). At such resolutions, it is very difficult to differentiate between the small-scale field generated by the tangling of the large-scale field, the small-scale dynamo action, and the compression of magnetic fields. Thus, at present, it is not possible to get a pure observational signature of magnetic fields generated by the small-scale dynamo in spiral galaxies.

The observations of magnetic fields in elliptical galaxies can serve as a probe of the small-scale dynamo action since the large-scale dynamo is inactive due to lack of large-scale differential rotation
(Seta et al. 2021). Type 1a supernova explosions from old stars and stellar winds (Moss \& Shukurov 1996; Mathews \& Brighenti 1997) drive turbulence in elliptical galaxies, which in turn amplifies magnetic fields by the small-scale dynamo action. For a typical elliptical galaxy, the study of Seta et al. (2021) estimated the driving scale of turbulence to be of the order of $300 \mathrm{pc}$, the root mean square (rms) turbulent velocity to be of the order of $2.5 \mathrm{~km} \mathrm{~s}^{-1}$, and the small-scale dynamo amplified magnetic field strength in the range $0.2-1 \mu \mathrm{G}$. Furthermore, they estimated the small-scale magnetic fields in a small sample (30 sources) of ellipticals using the Laing-Garrington effect (polarization asymmetry in jets hosted by radio galaxies with a central AGN component) and obtained magnetic fields in the range $0.1-6.0 \mu \mathrm{G}$. In this paper, we aim to obtain magnetic fields in ellipticals from a richer (210 sources) and more recent dataset of sources exhibiting the Laing-Garrington effect, which presumably traces the magnetic fields of the depolarizing medium, usually the halo of hot gas around the ellipticals and/or the intracluster medium (ICM) (Laing 1988; Garrington \& Conway 1991). Thus, we also use the statistical properties of polarized emission from extragalactic background radio sources when seen through intervening galaxies to estimate magnetic fields in ellipticals, primarily in their haloes or the circumgalactic medium (CGM), which is the gas around galaxies outside their disks but inside their virial radii. Typical virial radius scales are $~ 200-250 \mathrm{kpc}$ in spirals (Putman et al. 2012; Tumlinson et al. 2017).

The structure of the paper is as follows. In Sec. 2, we provide the details of the terminology used throughout the paper and discuss analysis techniques. In Sec. 3, we use the Laing-Garrington effect to estimate magnetic fields in ellipticals (data in Sec. 3.1, methodology in Sec. 3.2, and results in Sec. 3.3). The magnetic fields using polarized emission from background radio sources is presented in Sec. 4 (data in Sec. 4.1 and Sec. 4.2, analysis in Sec. 4.4, and results in Sec. 4.5). Finally, we summarize our results in Sec. 5. Standard cosmology (flat $\Lambda \mathrm{CDM}$ model) is used throughout the paper.

\section{ANALYSIS TECHNIQUES}

\subsection{Using the Laing-Garrington effect}

The observed polarization difference between the radiation from a jet and counterjet (jet facing away from the observer), usually referred to as the Laing-Garrington effect (Laing 1988; Garrington et al. 1988), can be used to estimate the magnetic field of the host galaxy. Along the observer's line of sight, radiation from the counterjet travels a longer distance through the host galaxy's medium than radiation from the jet, leading to a higher depolarization for the counterjet due to the random fluctuations in the Faraday rotation measures $\left(\sigma_{\mathrm{RM}}\right)$ present because of the small-scale magnetic fields of the galaxy. Apart from the magnetic fields, the $\sigma_{\mathrm{RM}}$, and depolarization in turn, depends on various other parameters such as the electron density $\left(n_{\mathrm{e}}\right)$, correlation length $\left(\ell_{b}\right)$, path length $(L)$, and redshift $(z)$ (details in Sec. 3.2). Assuming that the jet and counterjet have similar intrinsic polarization properties, the difference in the observed depolarization (or equivalently polarization) signal can be associated with the magnetic field of the halo or the CGM of the host galaxy in the regions along the line of sight. (e.g., Seta et al. 2021).

The synchrotron emission in ellipticals is from the central active galactic nucleus (AGN) and the ISM (due to cosmic ray electrons and magnetic fields) (Jiang et al. 2010; Crocker et al. 2011). The study of Nyland et al. (2017) shows that $1.4 \mathrm{GHz}$ radio emission in the host galaxy extends to $18 \mathrm{kpc}$ in extended radio galaxies (see 
their Sec. 7). In the majority of their galaxies, the radio emission is associated with star formation (SF) activity, and in some rare cases, the extended jets of an active nucleus. The radio galaxy (RG) catalog we adopt consists of radio observations from AGN jets, lobes, and hotspots that extend much beyond the host galaxy's environment (Vernstrom et al. 2019), having impact parameters mostly in hundreds of kpc. Thus, in extended radio galaxies (ERGs), the extent of SF activity is negligible near their usual halo extent of a few hundred $\mathrm{kpc}$, as well as for the large impact parameters we encounter in our sample of RGs. Ignoring the internal Faraday depolarization of the polarized emission from the AGN jets (or lobes, hotspots) in our sample of radio galaxies, it would be reasonable to assume that the polarization properties of this emission are not affected much by any other synchrotron emission (due to lack star formation at such great distances beyond the host galaxy) along the line of sight in the ERGs. For giant radio galaxies (GRGs) extending to a few Mpc, a very small fraction (12/820) show the potential for star formation (Dabhade et al. 2020). For this reason, polarized emission from radio galaxies do not have deep Faraday screens in general, unless the polarization is seen from the radio core associated with the host galaxy. Thus, depolarization in our sample of RGs mainly occurs in the medium in which the plane of polarization is only rotated by Faraday rotation due to thermal electrons and magnetic fields, and for this, the degree of polarization $p$ depends on the observing wavelength $(\lambda)$ and the standard deviation of rotation measure fluctuations $\left(\sigma_{\mathrm{RM}}\right)$ as (Burn 1966; Sokoloff et al. 1998)

$$
p=p_{0} \exp \left(-2 \sigma_{\mathrm{RM}}^{2} \lambda^{4}\right),
$$

where $p_{0}$ is the intrinsic degree of polarization.

Assuming that the intrinsic polarization properties of the jet and counterjet in an elliptical are similar, the ratio of the observed degree of jet and counterjet polarization, $p_{\mathrm{j}}$ and $p_{\mathrm{cj}}$, at the observing wavelength $\lambda$ is expressed as

$$
\frac{p_{\mathrm{j}}}{p_{\mathrm{cj}}}=\exp \left(-2 \lambda^{4}\left(\sigma_{\mathrm{RM}_{\mathrm{j}}}^{2}-\sigma_{\mathrm{RM}_{\mathrm{cj}}^{2}}^{2}\right)\right),
$$

where $\sigma_{\mathrm{RMj}}$ and $\sigma_{\mathrm{RMcj}}$ are the standard deviations of the rotation measure fluctuations for the jet and counterjet, respectively. For smaller physical separation between the lobes, the contribution from the galactic foreground (including the IGM) is similar (Vernstrom et al. 2019), and most likely cancels out on division. Using Eq. 4, we can estimate the standard deviation of the rotation measure fluctuations in the halo (or the circumgalactic medium) of the elliptical galaxy (also accounting for the redshift of the source now), $\sigma_{\mathrm{RMell}}$, as

$$
\sigma_{\mathrm{RMell}}=\frac{\left(\sigma_{\mathrm{RM}_{\mathrm{cj}}}^{2}-\sigma_{\mathrm{RM}_{\mathrm{j}}^{2}}\right)^{1 / 2}}{(1+z)^{2}} .
$$

We use this analysis ${ }^{1}$ for the data classified as physical pairs (210 sources) in Vernstrom et al. (2019) (V19) to compute $\sigma_{\text {RMell }}$ for those host galaxies' halo or CGM. Then, assuming two different thermal electron density distributions, we estimate magnetic field strengths from the computed $\sigma_{\mathrm{RM}}$ ell values (Sec. 3).

\footnotetext{
1 The study of Seta et al. (2021) uses the ratio of observed depolarization fraction for the jet and counterjet instead of the degree of polarization, which we use.
}

\subsection{Using the Faraday Rotation Measure (RM) from background radio sources}

The observed RM from an extragalactic radio source is actually a sum of contributions from all the intervening magneto-ionic regions between the source and us; thus, RM can be expressed as

$$
\mathrm{RM}=\mathrm{RM}_{\text {source }}+\mathrm{RM}_{\mathrm{IGM}}+\mathrm{RM}_{\text {ingal }}+\mathrm{RM}_{\mathrm{MW}},
$$

where $\mathrm{RM}_{\text {source }}$ is the $\mathrm{RM}$ arising due to the background polarized source (for example, radio lobes), $\mathrm{RM}_{\mathrm{IGM}}$ is the contribution from the foreground intergalactic medium, $\mathrm{RM}_{\text {ingal }}$ is the contribution from intervening galaxies (including the host galaxy of the background polarized source and excluding the Milky Way), and $\mathrm{RM}_{\mathrm{MW}}$ is the contribution from the Milky Way.

Magnetic fields in the IGM are of the order of $10^{-3} \mu \mathrm{G}$ (Grasso \& Rubinstein 2001; de Gouveia Dal Pino 2006), whereas the observed galactic magnetic fields are in the range $1-10 \mu \mathrm{G}$ (Beck 2015). The intergalactic thermal electron density $\left(\sim 1.6 \times 10^{-7} \mathrm{~cm}^{-3}\right.$, Yao et al. $2017)$ is also much smaller than average thermal electron density in spiral galaxies $\left(\sim 0.02 \mathrm{~cm}^{-3}\right.$, Berkhuijsen \& Müller 2008; Gaensler et al. 2008; Yao et al. 2017). Thus, even though the path length of the light traversing through the IGM is about a thousand times higher than that for the galaxies (approximately kpc for galaxies and Mpc for the IGM), the contribution of the IGM to the observed RM, $\mathrm{RM}_{\mathrm{IGM}}$ in Eq. 6, can be safely neglected in comparison to the other terms. The Milky Way contribution, $\mathrm{RM}_{\mathrm{MW}}$, can be modeled using the Faraday rotation map of the Galactic sky, such as the Oppermann map (Oppermann et al. 2015), and the map by (Hutschenreuter et al. 2021) (referred as Faraday 2020 hereon with further details given in Sec. 4.1). Thus, the Milky Way part can be subtracted from the RM to obtain the residual $\mathrm{RM}$ contribution, $\mathrm{RRM}=\mathrm{RM}-\mathrm{RM}_{\mathrm{MW}}$, which would ideally have contributions from the source and the intervening galaxies only.

Given a distribution for $\mathrm{RM}_{\text {source, }}$ the RRM distribution would depend on the number and morphology of the intervening galaxies. For a fixed number of intervening galaxies, considering that the spiral galaxies have stronger magnetic fields and higher thermal electron density (Taylor \& Cordes 1993) than the ellipticals (Mathews \& Brighenti 2003), we expect that the standard deviation of RRM would be higher for intervening spirals than for intervening ellipticals. Furthermore, for a fixed morphology, we would expect that the standard deviation of RRM would increase with the number of intervening galaxies. We derive the statistical properties of RRM distribution, computed using the RM of background sources from the catalog compiled by Farnes et al. (2014a)) and the Milky Way RM model from Faraday 2020 for different morphologies (decided based on the Galaxy Zoo data, Lintott et al. 2008), to study their dependence on the number of intervening galaxies and morphology. From this, we finally estimate magnetic fields in elliptical galaxies (Sec. 4). The estimated magnetic field in galaxies from both methods is primarily from the halo or circumgalactic medium.

\subsection{Terminology}

In the list below, we define common terms which will be used throughout the paper.

- $\mu_{\text {sample }}:$ mean of a sample dataset

- $\sigma_{\text {sample }}:$ standard deviation of a sample dataset

- $\mathcal{S}_{\text {sample }}$ : skewness of a sample dataset

- $\mathcal{K}_{\text {sample }}$ : kurtosis of a sample dataset

- $\sigma_{\mathrm{RRM}}$ : standard deviation of the residual RMs, i.e., the RMs 
from multiple background sources with the Milky Way RM contribution removed

- $\sigma_{\mathrm{RM}}$ : standard deviation of fluctuation of rotation measures in a single host galaxy

- $\mathrm{R}_{\mathrm{gal}}$ : radius of the galaxy (assumed to be spherical)

- $\mathrm{R}_{\text {halo/CGM }}$ : radius of the galaxy's halo or CGM (10 $\left.\mathrm{R}_{\mathrm{gal}}\right)$ (assumed to be spherical)

- $\mathrm{r}_{\perp}$ : impact parameter (distance from galaxy's center at which the light from the background source passes)

- ip $_{\text {ratio }}: r_{\perp} / R_{\text {gal }}$

- $N_{\text {ingal }}$ : number of intervening galaxies through which the light from the background source passes before reaching the observer.

\section{MAGNETIC FIELDS IN ELLIPTICAL GALAXIES USING THE LAING-GARRINGTON EFFECT}

\subsection{Radio Galaxy (RG) Catalog}

Recently, Vernstrom et al. (2019) (referred as V19 hereon) presented a study of the classification of RM sources from the NVSS catalog (Taylor et al. 2009) into 317 physical pairs and 5111 random pairs, by making use of infrared images from Wide-Field Infrared Survey Explorer (WISE, Wright et al. (2010)), optical images from Sloan Digital Sky Survey (SDSS) data release 9 (Ahn et al. 2012), and the $1.4 \mathrm{GHz}$ images from the Faint Images of the Radio Sky at Twentycm (FIRST, Becker et al. (1995)). Physical pairs are tantamount to the lobes or hotspots of giant radio galaxies (GRGs) or extended radio galaxies (ERGs), where the lobes extend well beyond the host galaxy's local environment into the CGM or surrounding IGM/ICM. The two lobes are probably jet (closer to us) and counterjet (facing away from us) side. However, a physical pair could also be composed of radio hotspots instead of radio lobes.

The angular separation between the lobes, polarization values and off-axis leakage polarization for each lobe, the redshift of the sources, and the difference in the RM of two lobes, $\Delta \mathrm{RM}$, with the corresponding error, $\Delta \mathrm{RM}_{\text {error }}$, were accessed and utilized for our analysis ${ }^{2}$. We only retain 210 physical pairs for which the redshifts are available. Together with their angular separation and the redshift $z$, we determine the distance between the polarized components of the lobes (physical separation) for which the RM and polarization values are available $^{3}$. Our RG catalog consists of 91 RGs with physical separation less than $500 \mathrm{kpc}, 85 \mathrm{RGs}$ between $500 \mathrm{kpc}-1 \mathrm{Mpc}, 27 \mathrm{RGs}$ between $1-2 \mathrm{Mpc}$, and $7 \mathrm{RGs}$ between $2-4 \mathrm{Mpc}$. Due to the lack of inclination angle data, we do not account for the bent tail type radio galaxies and assume that the angle between the lobes is $180^{\circ}$. Additionally, the angle between the lobes' orientation and the line of sight is not known from the data. Thus, the distance between the lobes is the projection of distance in the observer's plane. This might lead to inaccurate measurements at the extremities (infrequent possibility) in lobe orientation, i.e., lobes oriented $\perp$ or $\|$ to the observer's plane.

The polarization values in V19 are taken from the NVSS cata$\log$, and percent polarization is the ratio of average peak polarized intensity to the peak integrated Stokes I (Taylor et al. 2009), which gives the lower limit to the true observed polarization. The average and integration are done with images at frequencies 1365 and 1435

\footnotetext{
2 The data is available at https://iopscience.iop.org/0004-637X/ 878/2/92/suppdata/apjab1f83t1_mrt.txt.

3 Astropy.cosmology.LambdaCDM was used to calculate the angular diameter distance to a body using its redshift.
}

MHz. Thus, the equivalent frequency for the average polarization values is $1400 \mathrm{MHz}$ (to be used for Eq. 3 and Eq. 4).

\subsection{Methodology to extract small- and large- scale magnetic field strengths using the RG catalog}

Here, we use the techniques described in Sec. 2.1 and the observed polarization properties of physical pairs with redshifts from the dataset in V19 to estimate magnetic fields in those radio galaxies. Radio galaxies are usually associated with ellipticals, and several observational results suggest that the majority of giant radio galaxies are giant ellipticals (Lilly \& Prestage 1987; Owen \& Laing 1989; Hamilton et al. 2000; Ho 2009; Saripalli 2012; Malarecki et al. 2013; Banfield et al. 2015). Furthermore, Garrington \& Conway (1991) attributed the asymmetry in depolarization of two jets to an external X-ray halo of ionized gas, which is often found in clusters. To ensure that the depolarization occurs inside the galaxy medium rather than the cluster, we cross-match the physical pairs of V19 with the SDSS catalog (Sec. 4.2), using the methodology described in Sec. 4.3, to find the host galaxies of the jets. We find 40 matches in the SDSS catalog, for which the diameter of the halo of the galaxy is considered ten times the diameter of the galaxy (see the last paragraph of Sec. 4.2). The depolarization is assumed to be from the galaxy medium if the halo diameter is greater than the lobes' physical separation; 26/40 RGs satisfy this condition. The average physical separation between the lobes for these $26 \mathrm{RGs}$ is $\sim 300 \mathrm{kpc}$ and is $\sim 600 \mathrm{kpc}$ for the rest 14 RGs. Based on this, we now consider only RGs with physical separation less than $500 \mathrm{kpc}$ for a greater probability of depolarization occurring inside the galaxy's medium. Typical CGM diameter in spirals is $\sim 400-500 \mathrm{kpc}$ (Putman et al. 2012; Tumlinson et al. 2017; Pakmor et al. 2020). Assuming a similar CGM diameter in ellipticals, the physical separation cap at $500 \mathrm{kpc}$ is consistent with CGM/halo scales (Gerhard 2010). Hence, we assume that the asymmetry in the depolarization arises mainly from the X-ray halo and CGM of an elliptical galaxy, as the available data has path lengths in hundreds of kpc (Tumlinson et al. 2017) from the galaxy's center, beyond which the depolarization effects can be safely neglected due to negligible thermal electron density and magnetic fields.

Using Eq. 4 and Eq. 5 for the data of physical pairs in V19, we obtain the standard deviation of rotation measure fluctuations in the host elliptical galaxies, $\sigma_{\text {RMell }}$, for 84 sources (after imposing $500 \mathrm{kpc}$ physical separation cap and removing galaxies with SDSS halo diameters smaller than the physical separation). $\sigma_{\text {RMell }}$, assuming a profile for thermal electron density, can be used to compute the root mean square (rms) strength of the small-scale random magnetic field in the elliptical galaxy. For thermal electron density distribution, we use two profiles: a uniform density profile for simplicity (Seta 2019) and a King profile (Sarazin \& White 1988; Felten 1996; Mathews \& Brighenti 2003).

For a uniform thermal electron density $\left(\overline{n_{\mathrm{e}}}=\right.$ constant $)$, the $\sigma_{\text {RMell }}$ can be expressed as

$$
\frac{\sigma_{\mathrm{RMell}}}{(1+z)^{2}}=\frac{(2 \pi)^{1 / 4}}{3^{1 / 2}} \mathrm{~K} \overline{n_{\mathrm{e}}} b_{\mathrm{rms}}\left(L \ell_{b}\right)^{1 / 2},
$$

where $z$ is the redshift, $\mathrm{K}=0.81 \mu \mathrm{G}^{-1} \mathrm{~cm}^{3} \mathrm{pc}^{-1} \mathrm{rad} / \mathrm{m}^{2}$ is a constant, and $L$ is the path length in pc (distance between the lobes, see Sec. 3.1). Assuming the correlation length of small-scale magnetic fields $\ell_{b}$ to be $100 \mathrm{pc}$ (see Sec. 5.3.1 in Seta 2019) and the average thermal electron density in the halo/CGM of an elliptical galaxy $\overline{n_{\mathrm{e}}}$ to be $0.01 \mathrm{~cm}^{-3}, b_{\text {rms }}$ can be estimated. The correlation length of small-scale magnetic field $\ell_{b}$ (assumed to be $100 \mathrm{pc}$ ) is $3-4$ times 
smaller (see Tab 2.1 in Seta 2019) than the driving scale of turbulence, 300 pc (see Sec. 5.2 in Seta 2019, for full derivation). Here, the turbulence is assumed to be driven by Type 1a supernovae explosions. This is evident from the observations of iron abundances (Mathews \& Brighenti 2003) and simulations of multiphase gas in elliptical galaxies (Li et al. 2020a,b).

For a King profile, $n_{\mathrm{e}}=n_{\mathrm{e}}(0)\left(1+(r / a)^{2}\right)^{-3 \beta / 2}$, where $n_{\mathrm{e}}(0)$ is the thermal electron density at $r=0$, and $a$ is the core radius, the $\sigma_{\mathrm{RMell}}$ close to the center $(r=0)$ is expressed as

$\frac{\sigma_{\mathrm{RMell}}(0)}{(1+z)^{2}}=\frac{(2 \pi)^{1 / 4}}{3^{1 / 2}} \mathrm{~K} n_{\mathrm{e}}(0) b_{\mathrm{rms}}\left(a \ell_{b}\right)^{1 / 2}\left(\frac{\Gamma\left(\frac{3}{2}(\gamma+1)-0.5\right)}{\Gamma\left(\frac{3}{2}(\gamma+1)\right)}\right)^{1 / 2}$,

where $\beta$ is assumed to be $1 / 2$ (following the gas distribution, see Matthaeus et al. 2003), and $\gamma=2 / 3$ (inspired by the flux freezing or cooling flow constraint, which gives $b_{\text {rms }} \propto \rho^{2 / 3}$ ). Now, the $\sigma_{\text {RMell }}$ at any radius $r$ (on the plane of the sky) can be estimated as

$$
\sigma_{\mathrm{RMell}}(r)=\frac{\sigma_{\mathrm{RMell}}(0)}{(1+z)^{2}}\left[1+\left(\frac{r}{a}\right)^{2}\right]^{-\left(\frac{1}{4} \Gamma\left(\frac{3}{2}(\gamma+1)-1\right)\right)} .
$$

Eq. 8 contains the contribution of varying $n_{\mathrm{e}}$ and $b_{\text {rms }}$, derived via integration of $\sigma_{\mathrm{RM}}$ (see Felten (1996) and Appendix A in Bhat \& Subramanian (2013)). To estimate small-scale rms magnetic field strengths, $b_{\text {rms }}$, using the King profile, we assume $n_{\mathrm{e}}(0)=0.1 \mathrm{~cm}^{-3}$ (Mathews \& Brighenti 2003), $\ell_{b}=100$ pc (as in the uniform thermal electron density case), and $a=3 \mathrm{kpc}$ (Garrington \& Conway 1991). We consider the calculated $\sigma_{\mathrm{RM}}$ frl from Eq. 5 to be equal to $\sigma_{\mathrm{RMell}}(L / 2)$ from Eq. 9 , where $L / 2$ is the approximate distance from the center in the plane of observation where the extra depolarized hotspot is assumed to be located (since $L$ is the physical separation in the plane of observation). As Eq. 9 predicts the $\sigma_{\text {RMell }}$ at a distance from the center in the plane of observation along the line of sight, it is applicable at $L / 2$, the location of the extra depolarized hotspot (presumably counterjet).

Besides using the standard deviation of rotation measure fluctuations, $\sigma_{\mathrm{RMell}}$, to estimate the rms strength of small-scale magnetic fields (using Eq. 7 or Eq. 8 and Eq. 9), we also use the observed RM values from the physical pairs data in V19 to estimate the strength of large-scale magnetic fields.

RM (Eq. 2) can be approximated in terms of mean quantities as

$$
\mathrm{RM}_{\mathrm{j}, \mathrm{cj}} \approx \frac{\mathrm{K} \overline{n_{\mathrm{e}}} \overline{B_{0 \|}} L_{\mathrm{j}, \mathrm{cj}}}{(1+z)^{2}}
$$

where $\overline{B_{0 \|}}$ is the ordered parallel component of the large-scale magnetic field, and $L_{\mathrm{j}, \mathrm{cj}}$ is the path length of jet and counterjet, respectively. Note that we assume the elliptical galaxy to be a single and homogeneous Faraday rotating medium to calculate the large-scale fields $\left(\overline{B_{0 \|}}\right)$ along the line of sight. We assume that the difference in the $R M$ values from the two lobes (jet, $R M_{j}$, and counterjet, $R M_{c j}$ ) of the radio galaxy, $\Delta \mathrm{RM}$, is primarily due to different distances traversed by the radiation and can be expressed as

$$
\Delta \mathrm{RM} \approx \frac{\mathrm{K} \overline{n_{\mathrm{e}}} \overline{B_{0 \|}} L}{(1+z)^{2}},
$$

where $L$ is the physical separation between the two lobes in pc. Assuming $\overline{n_{\mathrm{e}}}=0.01 \mathrm{~cm}^{-3}$ and using $\triangle \mathrm{RM}$ and $L$ from the data, $\overline{B_{0 \|}}$ can be estimated.

It is important to reiterate that $L$ in Eq. 7 and Eq. 11 is taken to be equal to the distance or physical separation between the two

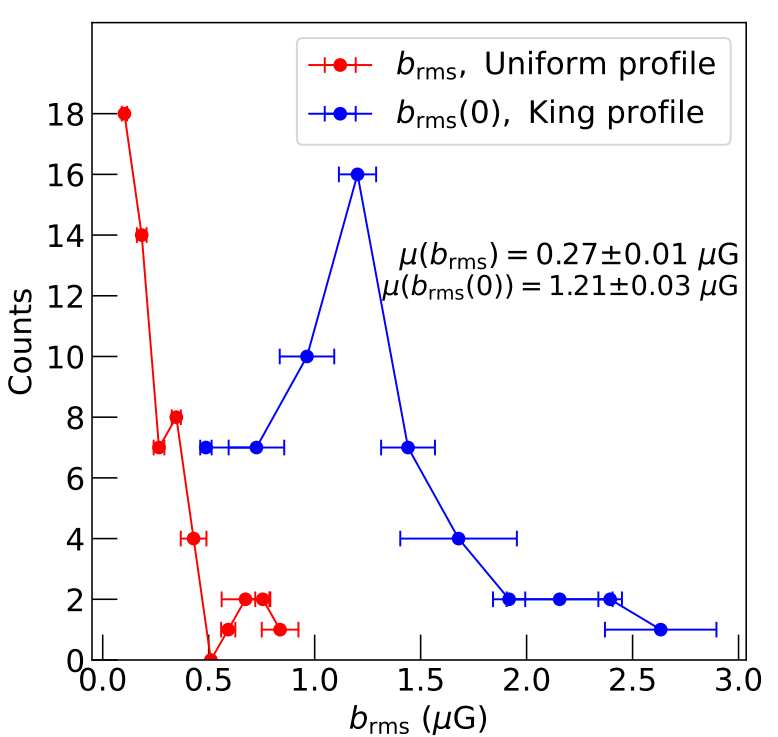

Figure 1. Histogram of rms strengths of small-scale magnetic fields ( $b_{\mathrm{rms}}$ for the uniform profile and $b_{\mathrm{rms}}(0)$ for the King profile) estimated using the Laing-Garrington effect in the RG sample ( $~ 57$ sources). The errors of the bins are standard deviations of the variation of the mean of every point in a bin calculated by the bootstrapping method with 500 iterations. The magnetic field strength for both electron density profiles lies in the range $0.06-2.75 \mu \mathrm{G}$.

radio lobes or hotspots in pc. This means that the $\overline{B_{0 \|}}$ estimated from Eq. 11 will be averaged over the path length (hundreds of $\mathrm{kpc}$ ) scales. Hence, the large-scale fields extracted from this analysis will be ordered over similar scales, i.e., hundreds of kpc. This also ensures that the effect of small-scale random magnetic fields on RM cancels out on averaging over such large-length scales since the correlation length of the small-scale field is comparatively quite small, i.e., of the order of $100 \mathrm{pc}$.

\subsection{Results from the analysis of the RG catalog using the Laing-Garrington effect}

After including the RGs with physical separation between the lobes less than $500 \mathrm{kpc}$, and removing the galaxies with SDSS halo diameters smaller than the physical separation $(<500 \mathrm{kpc}$, SDSS detected), we retain 84 RGs for which depolarization probably occurs in the galaxy's medium. The $b_{\text {rms }}$ (assuming uniform thermal electron density, Eq. 7), $b_{\text {rms }}(0)$ (assuming a King profile for the thermal electron density, Eq. 8), and $\overline{B_{0 \|}}$ (Eq. 11) are calculated for 84/210 RGs available from the physical pairs in V19. However, after considering errors in $b_{\text {rms }}$ values due to off-axis leakage polarization (see Ma et al. 2019, for further details), roughly 57 RGs are retained with the magnitude of $b_{\text {rms }}$ and $b_{\text {rms }}(0)$ being twice their respective errors. Fig. 1 shows the histogram of $b_{\text {rms }}$ and $b_{\text {rms }}(0)$ for the RG sample and the rms field strengths lies in the range $0.06-2.75 \mu \mathrm{G}$. The mean of $b_{\mathrm{rms}}$ is $0.27 \pm 0.01 \mu \mathrm{G}$ and that of $b_{\mathrm{rms}}(0)$ (naturally expected to be slightly higher because it only probes the magnetic field strength in the core of the elliptical galaxy) is $1.21 \pm 0.03 \mu \mathrm{G}$. For the whole sample, the estimated rms small-scale magnetic field strength in ellipticals $(\lesssim 3 \mu \mathrm{G})$ is much smaller than that of the Milky 

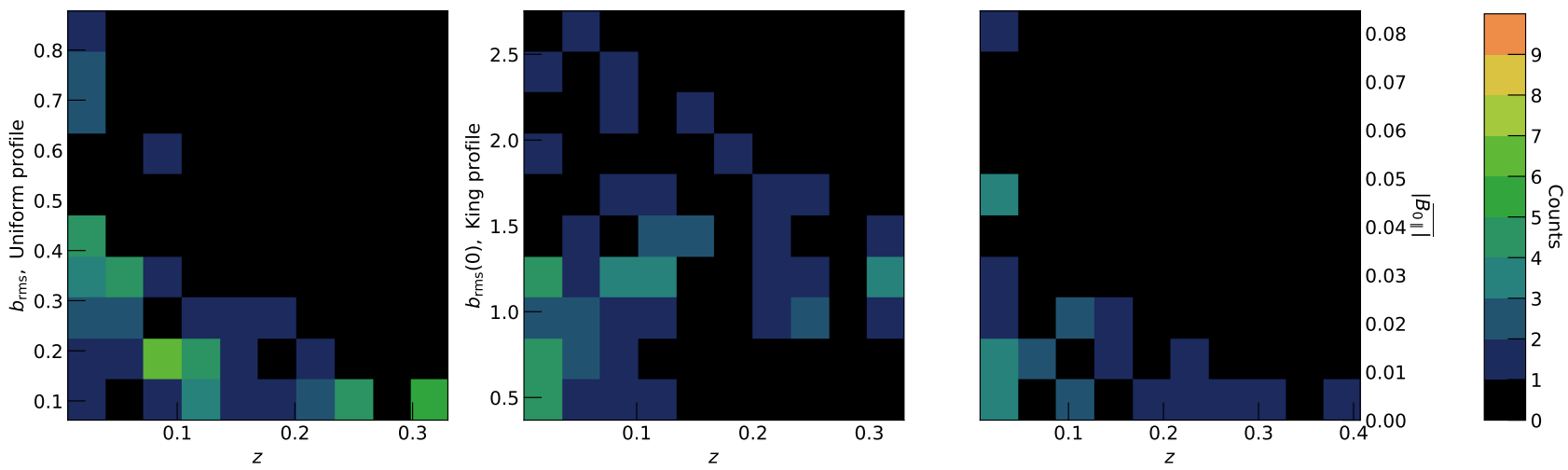

Figure 2. Two-dimensional histogram of the rms small-scale magnetic field strengths (left, middle plots), and large-scale magnetic field strengths (right plot), as a function of redshift with colors showing the counts for all the cases. The unit of magnetic fields is $\mu \mathrm{G}$. The counts reduce with redshift in both the rms small-scale field plots; however, the $b_{\text {rms }}(0)$ (King profile) seems to have an increasing trend with the redshift as opposed to $b_{\text {rms }}$ (uniform profile). The $b_{\text {rms }}(0)$ somewhat exhibits the trend expected for small-scale magnetic fields in cosmological simulations shown in Fig. 1 of Seta et al. (2021). Older galaxies (high $z$ ) tend to have more gas and a more turbulent medium than the younger ones (low $z$ ), implying that their magnetic field strengths will be higher due to the small-scale dynamo action. The large-scale field is an order of magnitude lower than the small-scale field and fluctuates around zero with redshift (we plot only its absolute values). However, the dispersion of large-scale field (right plot) seems to reduce with redshift. This might be possible due to a low number of RGs at higher $z$.
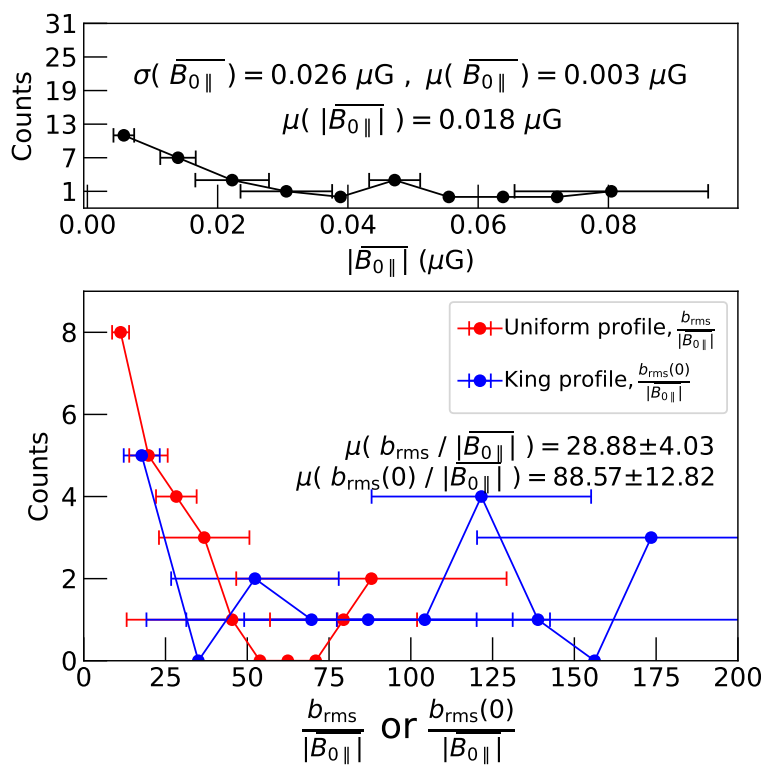

Figure 3. Top: Histogram for the estimated large-scale magnetic field strengths, $\overline{B_{0 \|}}$, for 26 sources. The errors of the bins are standard deviations of the variation of the mean of every point in a bin calculated by the bootstrapping method with 500 iterations. The mean and standard deviation are much smaller than the average $b_{\text {rms }}$ or $b_{\text {rms }}(0)$ (see Fig. 1). This shows that the small-scale magnetic fields in ellipticals are much stronger than the large-scale fields. Bottom: Histogram of the ratio of the small-to-large scale field strengths for $\sim 20$ RGs in the sample. Errors calculated are high at higher ratio values due to relatively large $\overline{B_{0 \|}}$ errors at such magnitudes. The small-scale magnetic field strengths can be as high as $25-90$ times stronger than the large-scale magnetic fields.

Way and nearby spiral galaxies, which is observed to be in the range $5-10 \mu \mathrm{G}$ (Beck 2015).

In Fig. 2, we show the two-dimensional histogram of the rms smallscale magnetic field strengths with redshift (in the range $0.01-0.46$ ) for both the uniform and King thermal electron density profiles, and the large-scale magnetic field strengths. For the uniform thermal electron density profile (left-panel of Fig. 2), as the redshift increases, the number of elliptical galaxies decreases, and the magnetic field strength does not seem to vary much beyond redshifts of 0.1. However, for the King profile case (right-panel of Fig. 2), the field strength seemingly increases with redshift even though the number of ellipticals decreases. The trend with redshift seen for the King profile case roughly agrees with that obtained from the the cosmological simulations (see Fig. 1 in Seta et al. 2021). As the redshift increases, the turbulence and amount of gas both increase, enhancing small-scale magnetic fields. However, a sample with inadequacies in data at higher redshifts could also generate a similar trend; hence, more robust datasets are required to confirm this trend. We see that the large-scale fields fluctuate roughly around zero, and the dispersion increases as the redshift decreases. This might be because of a different number of sources at different redshifts.

$\overline{B_{0 \|}}$, the large-scale field component of the radio galaxies, is estimated using Eq. 11 with corresponding errors calculated by propagating RM uncertainties. 26 RGs are retained for which the error in $\overline{B_{0 \|}}$ is less than half of the value. The top panel of Fig. 3 shows the histogram of $\left|\overline{B_{0 \|}}\right|$ and the computed absolute mean and standard deviation of the $\overline{B_{0 \|}}$ distribution $(0.018 \mu \mathrm{G}$ and $0.026 \mu \mathrm{G}$, respectively) are much smaller than small-scale field strengths (Fig. 1). The insignificant value of the mean of $\overline{B_{0 \|}}(0.003 \mu \mathrm{G})$ might be because of averaging over $\mathrm{kpc}$ scales and/or changing orientation (positive or negative) of the line of sight of the large-scale field. However, the standard deviation and the absolute mean of the $\overline{B_{0 \|}}$ distribution are also very small compared to the rms small-scale field strengths.

Furthermore, for approximately 20 radio galaxies (after removing the ratios for which the error is greater than the value), for which both the small-scale rms field strengths and $\overline{B_{0 \|}}$ values were available, the bottom panel of Fig. 3 shows the ratio of small-to-large scale field strengths (limited to a maximum value of 200 to prevent extremely high ratios due to negligible large-scale field strengths; only 2 points were removed as a result of this condition). All the reported errors were calculated by propagating the errors obtained from observations. The rms small-scale field strengths, for both the uniform and 
Table 1. This table is a representative of the given and extracted values from the data of V19 for depolarization by galaxies (physical separation between the lobes less than $500 \mathrm{kpc}$ ). The columns are as follows: column1: V19 source identifier number, column2: redshift of the source, column3: path length or projected physical separation between the lobes, column4: diameter of the halo of the host galaxy of the radio lobes (of V19) identified in SDSS by cross-matching, column5: ratio of the polarization values of the jet and counterjet, column6: calculated standard deviation of rotation measure in elliptical's CGM/halo, column7: small-scale magnetic fields with their one-sigma uncertainties for the uniform $n_{\mathrm{e}}$ case, column8: small-scale magnetic fields with their one-sigma uncertainties at the center of ellipticals for the King profile $n_{\mathrm{e}}$ case, column9: large-scale magnetic fields, column10: ratio of small-to-large scale magnetic fields for the uniform $n_{\mathrm{e}}$ case, column11: ratio of small-to-large scale magnetic fields for the King profile case. The dashes represent the lack of values for the corresponding parameter due to a failure in meeting the error conditions. The complete table is available in the supplementary text.

\begin{tabular}{|c|c|c|c|c|c|c|c|c|c|c|}
\hline V19 no. & $z$ & $\begin{array}{c}L \\
(\mathrm{kpc})\end{array}$ & $\begin{array}{c}\text { SDSS } \\
\text { identified } \\
\text { host size } \\
(\mathrm{kpc})\end{array}$ & $p_{\mathrm{j}} / p_{\mathrm{cj}}$ & $\begin{array}{c}\sigma_{\mathrm{RM}} \\
\left(\mathrm{rad} / \mathrm{m}^{2}\right)\end{array}$ & $\begin{array}{l}b_{\text {rms }} \\
(\mu \mathrm{G})\end{array}$ & $\begin{array}{c}b_{\mathrm{rms}}(0) \\
(\mu \mathrm{G})\end{array}$ & $\begin{array}{l}\overline{\overline{B_{0 \|}}} \\
(\mu \mathrm{G})\end{array}$ & $\overline{b_{\mathrm{rms}}} /\left|\overline{\boldsymbol{B}_{0 \|}}\right|$ & $\overline{b_{\mathrm{rms}}(0) /\left|\overline{B_{0 \|}}\right|}$ \\
\hline 38 & 0.097 & 174 & - & 1.29 & 6.53 & $0.18 \pm 0.03$ & $0.82 \pm 0.15$ & - & - & - \\
\hline 142 & 0.098 & 208 & 293 & 1.53 & 8.34 & $0.2 \pm 0.02$ & $1.14 \pm 0.11$ & - & - & - \\
\hline 438 & 0.03 & 384 & - & 1.18 & 5.9 & $0.12 \pm 0.02$ & $1.06 \pm 0.19$ & $0.0061 \pm 0.0007$ & $19.97 \pm 5.99$ & $174.67 \pm 52.39$ \\
\hline 521 & 0.3 & 431 & - & 1.04 & 1.82 & - & - & - & - & - \\
\hline 1654 & 0.133 & 257 & 2000 & 1.34 & 6.51 & $0.14 \pm 0.02$ & $0.98 \pm 0.13$ & - & - & - \\
\hline 2143 & 0.128 & 235 & 321 & 1.22 & 5.37 & - & - & - & - & - \\
\hline 3378 & 0.013 & 183 & - & 2.64 & 14.83 & $0.46 \pm 0.03$ & $1.92 \pm 0.12$ & $0.0177 \pm 0.0031$ & $25.73 \pm 6.08$ & $108.01 \pm 25.53$ \\
\hline 3458 & 0.129 & 209 & - & 1.21 & 5.27 & - & - & - & - & - \\
\hline 3892 & 0.095 & 160 & - & 1.43 & 7.74 & $0.22 \pm 0.1$ & $0.94 \pm 0.42$ & - & - & - \\
\hline 4451 & 0.242 & 369 & - & 1.66 & 7.12 & $0.1 \pm 0.02$ & $1.26 \pm 0.22$ & $-0.0111 \pm 0.0046$ & $9.26 \pm 5.51$ & $113.44 \pm 67.47$ \\
\hline
\end{tabular}

King thermal electron density profile, are significantly higher (mean of the ratios are $28.88 \pm 4.03$ and $88.57 \pm 12.82$ for uniform and King profile cases, respectively) than that of the large-scale fields. The same analysis, when performed with three-sigma accuracy, yields very similar results with $b_{\text {rms }}$ in the range $0.06-2.75 \mu \mathrm{G}$ and mean of the small-to-large scale field ratios in the range 25-70. These ratios are much larger in ellipticals as compared to spirals, where the ratio of small to large scale field strengths lie in the range $1-5$ (Fletcher et al. 2011; Beck 2015). Thus, the small-scale field generation mechanisms are probably much more efficient in our sample of ellipticals as compared to the large-scale dynamo. This is probably because the large-scale field dynamo is inactive due to a lack of significant rotation in the ellipticals (Beck et al. 1996; Moss \& Shukurov 1996). However, due to the small number of available radio galaxies for which both the small- and large-scale fields can be estimated ( 20 out of 210 galaxies), and large error bars in the bottom plot of Fig. 3, better statistics are needed to confirm that the small-scale field generation mechanisms are dominant.

\section{USING BACKGROUND POLARIZED RADIO SOURCES}

\subsection{RM catalog}

We use redshift, RM, and polarization data for all 4003 background polarized sources from Farnes et al. (2014a) for our study (they obtained the RM values from Taylor et al. (2009) and redshifts by cross-matching the original NVSS data set in Condon et al. (1998) to a catalog of NVSS RM redshifts in Hammond et al. (2012)). The dataset is expected to contain mostly the jet-dominated sources in classical radio galaxies and quasars (see Sec. 7 of Farnes et al. 2014a). These RMs also have Milky Way contribution, $\mathrm{RM}_{\mathrm{MW}}$, which we remove using the data in Oppermann et al. (2015) or Faraday $2020{ }^{4}$. Throughout the paper (except in Fig. 4 where we show a comparison between both the maps), we use Faraday 2020. We use RING-ordered

4 The Milky Way RM data is available at https://wwwmpa. mpa-garching.mpg.de/ift/faraday/2014/index.html and https://wwwmpa.mpa-garching.mpg.de/ ensslin/research/ data/faraday $2020 . \mathrm{html}$
HEALPix maps at resolution $N_{\text {side }}=512$ to extract $\mathrm{RM}_{\mathrm{MW}}$ for 3145728 pixels across the whole sky. The angular resolution of each pixel is around $0.1 \mathrm{deg}^{5}$. Based on the coordinates of the background RM source, the closest pixel number from the HEALPix map was calculated, and the Galactic rotation measure $\left(\mathrm{RM}_{\mathrm{MW}}\right)$ for that pixel was subtracted from the background (extragalactic) RM data to obtain the residual rotation measure, RRM.

The top panel of Fig. 4 shows RM with and without subtracting the Milky Way contribution as a function of the Galactic latitude, b, and the bottom panel shows their standard deviation over Galactic latitude bins of size $5^{\circ}$. We would expect the standard deviation of residual contributions to remain nearly uniform throughout the sky as they only represent the background source distribution. However, there is some dependence of the standard deviation of RRM on the Galactic latitude, especially in the range $-30^{\circ}<\mathrm{b}<30^{\circ}$. This is probably due to incomplete Milky Way subtraction, despite correcting with the model from Oppermann et al. (2015) and Faraday 2020. Thus, for our study, to avoid any unintended Milky Way contribution, all the background RM sources appearing inside the Galactic latitude range $-30^{\circ}<\mathrm{b}<30^{\circ}$ were removed. As a result, only 3098 of 4003 points were preserved outside the $\pm 30^{\circ}$ of the Galactic plane.

The NVSS rms resolution for the coordinates of the faintest radio sources (flux densities < $15 \mathrm{mJy}$, only 227 out of a total of 37000 sources) is $7 \operatorname{arcsec}^{6}$. This is about $60 \mathrm{kpc}$ at $z=1$, significantly lesser than the average $\mathrm{R}_{\text {halo/CGM }}$ of the galaxies in our sample (about $500 \mathrm{kpc}$, see Sec. 4.5.2). Additionally, for the rest of the majority of the sources, rms resolution is less than 1 arcsec. This is equivalent to a distance of less than $10 \mathrm{kpc}$ at $z=1$, which is small enough to prevent any false detection of the intervening galaxies.

\subsection{Galaxy Catalog}

To explore the effects of intervening galaxies on RM from the background polarized sources, we use the galaxy catalog provided by the 16th data release from the SDSS (Ahumada et al. 2020). It is the

\footnotetext{
5 https://lambda.gsfc.nasa.gov/toolbox/tb_pixelcoords.cfm

6 https://www.cv.nrao.edu/nvss/
} 


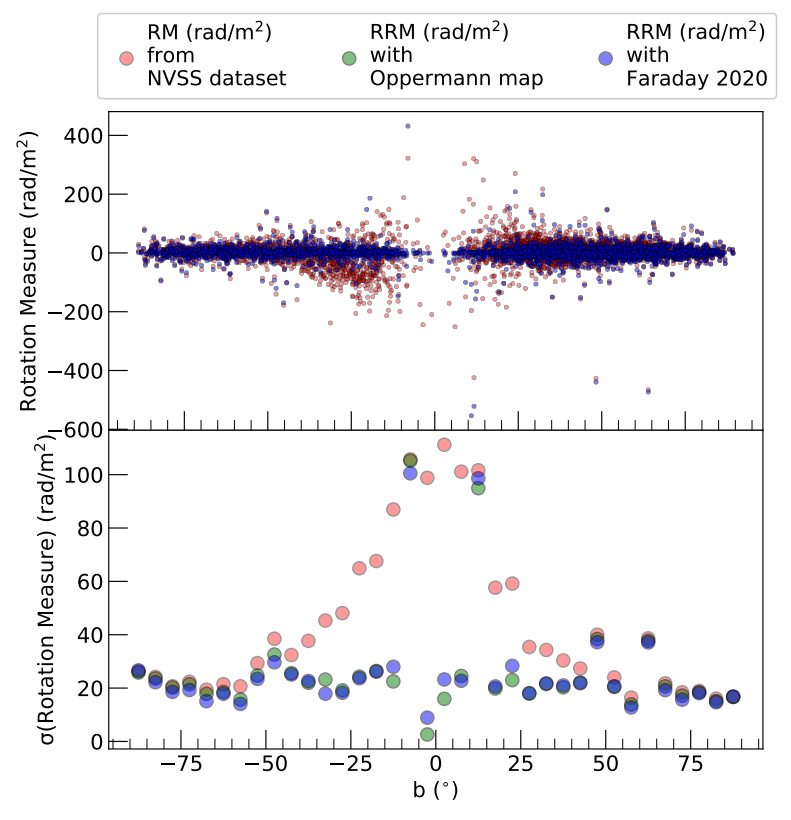

Figure 4. Top: RM (from Farnes et al. (2014a)) and RRM (from Farnes et al. (2014a) with the Milky Way contribution removed using Faraday 2020) as a function of galactic latitude, b. Bottom: Dispersions of rotation measures when binned into 36 equidistant bins as a function of Galactic latitude. $\sigma$ of RRM with Milky Way subtraction from Oppermann map (Oppermann et al. 2015, green dots), and Faraday 2020 (Hutschenreuter et al. 2021, blue dots) is shown. Due to a low number of RM and RRM values available in the bins near $b=0^{\circ}$, the $\sigma$ (Rotation Measure) takes unpredictable values, and we denote the dip at the center of this graph for the corrected case to a small number of RRM values in that bin. Since the RRM ideally represents only the extragalactic background source distribution, we would expect the standard deviation of RRM to be flat with the Galactic latitude. However, $\sigma(\mathrm{RRM})$ is seen to be high in some bins within $-30^{\circ}<\mathrm{b}<30^{\circ}$ and this might be due to incomplete removal of the Milky Way contribution (especially the largescale gradients). Thus, we only select sources beyond $\pm 30^{\circ}$ of the Galactic latitude. For all the subsequent analyses in the study, we adopt Faraday 2020 to estimate the Milky Way RM contribution and finally calculate the residual rotation measure (RRM).

fourth data release from SDSS-IV (following DR13, DR14, DR15). DR16 provides spectra along with spectroscopic redshifts for around 1 million unique galaxies, with the help of the eBOSS (Extended Baryon Oscillation Spectroscopic Survey, Dawson et al. 2016) ELG (Emission Line Galaxies) program and the data of APOGEE-2 South (Apache Point Observatory Galactic Evolution Experiment, Majewski et al. 2017). The SDSS catalogs were found and queried on the Catalog Archive Server (Thakar et al. 2008) ${ }^{7}$. These catalogs contain photometric and spectroscopic properties, along with their derived object parameters.

The following parameters were extracted for galaxies from the CasJobs server: Galaxy Coordinates, Petrosian radius in $\mathrm{u}, \mathrm{g}, \mathrm{r}, \mathrm{i}$, and $\mathrm{z}$ bands ${ }^{8}$, spectroscopic redshifts (robust upto $z=0.7$ ). Morphology classifications of galaxies with/without spectra from the Galaxy Zoo (Lintott et al. 2008) were also obtained. An object is classified as a galaxy only if both the spectroscopic and photometric information

\footnotetext{
7 https://skyserver.sdss.org/casjobs/

8 Details of Petrosian radius can be found at https://www.sdss.org/ dr12/algorithms/magnitudes/.
}

classifies it as one. The coordinates and the Petrosian radii are obtained from the photometric observations, whereas redshifts are obtained from the spectroscopic data. Photometric, spectroscopic data of the SDSS and the Galaxy Zoo were connected using a common unique SDSS identifier, specobjid.

Galaxy Zoo 1 project (Lintott et al. 2011) categorized a total of 738,175 galaxies from the SDSS DR6, $92 \%$ of which have spectra available in SDSS DR7. Votes from more than 100000 volunteers, corrected for weightage of every user, were used to report $p_{\text {ell }}$ and $p_{\text {spi }}$, which are the probabilities of a galaxy being an elliptical or a spiral, respectively. As this is a statistical study, it would be useful to have most of the galaxies classified, so we take $p=0.65$ to be the threshold to categorize a galaxy into a morphological type (probability at which the Galaxy Zoo project claims to have less than $10 \%$ classification error). A galaxy in the sample is categorized into one of the following four categories: 'Elliptical' (if $p_{\text {ell }}>0.65$ and $p_{\text {spi }}<0.35$ ), 'Spiral' (if $p_{\text {spi }}>0.65$ and $p_{\text {ell }}<0.35$ ), 'Not Sure' (if both the conditions above are not satisfied), and 'Not Available' (if the probabilities for a galaxy type are unavailable in the Zoo dataset).

The maximum of the five Petrosian radii in $\mathrm{u}, \mathrm{g}, \mathrm{r}, \mathrm{i}$, and $\mathrm{z}$ bands is selected as the galaxy's radius, referred to as $\mathrm{R}_{\text {gal }}$ throughout the text. The galaxy's contribution to RM is considered up to 10 times the galaxy radius, which likely traces the halo or CGM $\left(\mathrm{R}_{\text {halo } / \mathrm{CGM}}=\right.$ $10 \mathrm{R}_{\text {gal }}$ with a maximum of $1 \mathrm{Mpc}$ ). Giant elliptical haloes can extend up to $1 \mathrm{Mpc}$ (Uson et al. 1990) and the CGM up to a few hundred kpc (Tumlinson et al. 2017). Thus, $\mathrm{R}_{\text {halo/CGM }}$ can be the radius of the halo or the CGM.

\subsection{Cross-matching the catalogs}

Fig. 5(a) shows the available galaxies from SDSS DR16. Fig. 5(b) shows the RM data sources and the known morphology of galaxies according to the Galaxy Zoo project. In Fig. 5(b), visually, there is a significant overlap between the intervening galaxies and RM sources.

We use coordinates of background polarized sources with available RM values from the NVSS catalog and galaxies from the galaxy catalog (SDSS DR16 catalog), respectively, to cross-match and detect the intervening galaxies. These background polarized sources are mostly jet-dominated sources due to their steep spectrum (see Fig. 5 and Sec. 7 of Farnes et al. 2014a) and their contribution is considered part of the $\mathrm{RM}_{\text {source }}$ term in Eq. 6. For a particular source, the DR16 catalog is scanned to identify the intervening galaxies that contain the source within its $R_{\text {halo/CGM }}\left(10 R_{\text {gal }}\right.$, where $\mathrm{R}_{\mathrm{gal}}$ varies with the galaxy). Also, see the left panel of Fig. 11 in V19 for a schematic. To ensure that the galaxies intervene, only those with redshifts (minus two-sigma error) smaller than that of the RM source (plus two-sigma error) are chosen, with an additional relaxation of $z=0.0005$ which corresponds to $\sim 2 \mathrm{Mpc}$ for nominal redshifts. Thus, the redshift condition for the intervention is $z_{\text {gal }}-2 \operatorname{error}\left(z_{\text {gal }}\right)<z_{\mathrm{RM}}+2 \operatorname{error}\left(z_{\mathrm{RM}}\right)+0.0005$. This condition ensures that the host galaxies of the background sources are also identified as intervening galaxies, and the additional relaxation covers the possible difference between the redshifts of the host galaxy's center and the background polarized source jet. Galaxies from the SDSS catalog have robust spectroscopic redshifts upto $z=0.7$. Thus, when the redshift of the RM source is $>0.7$, there could be undetected intervening galaxies in its line of sight. Impact parameter values (distance between the intervening galaxy's center and the point where radiation from the background source penetrates that galaxy) are also calculated using the coordinates. 


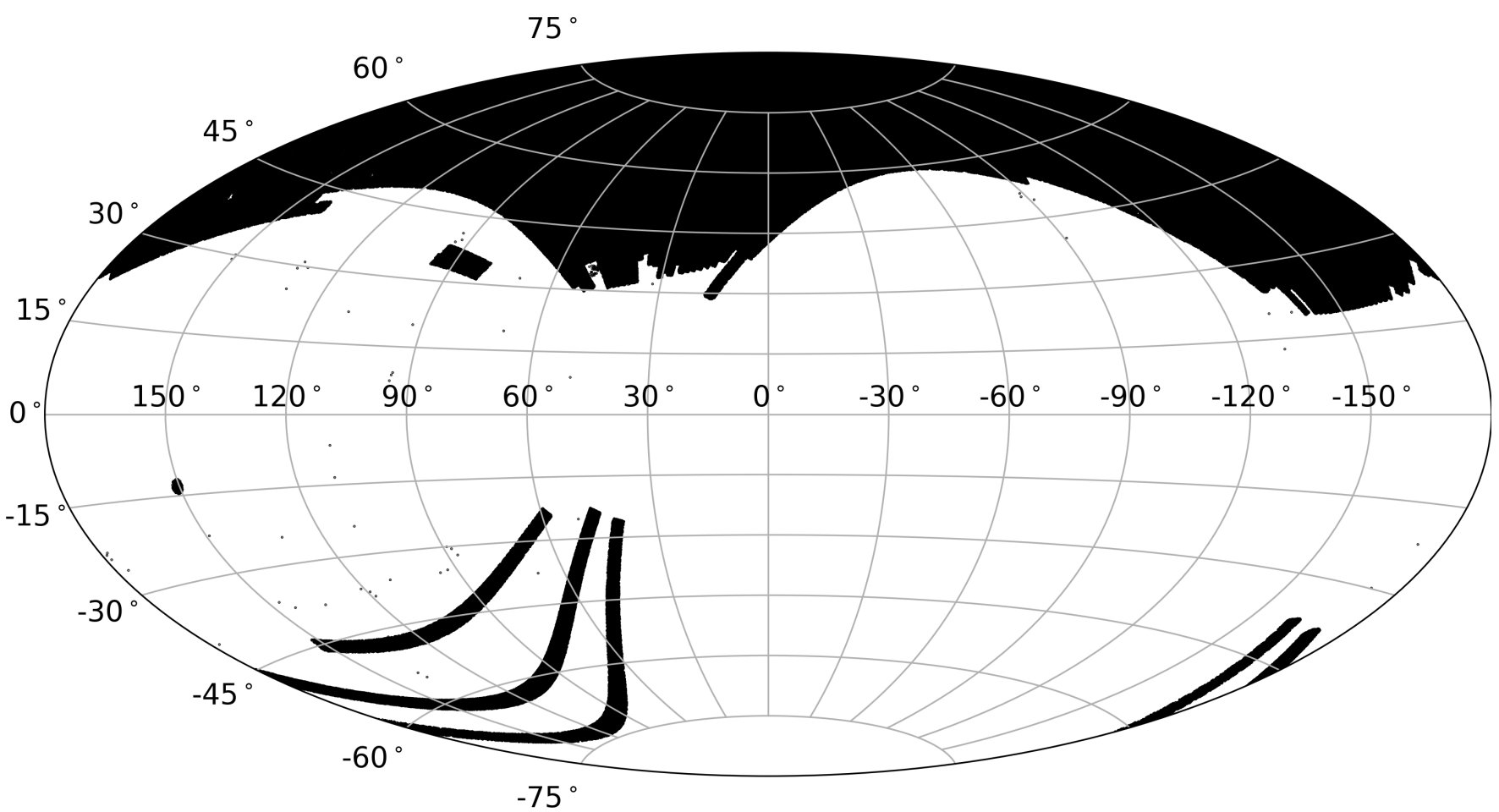

(a) Shaded parts are the regions covered by galaxies from the SDSS DR16 catalog. The figure contains approximately 0.9 million galaxies.

$75^{\circ}$

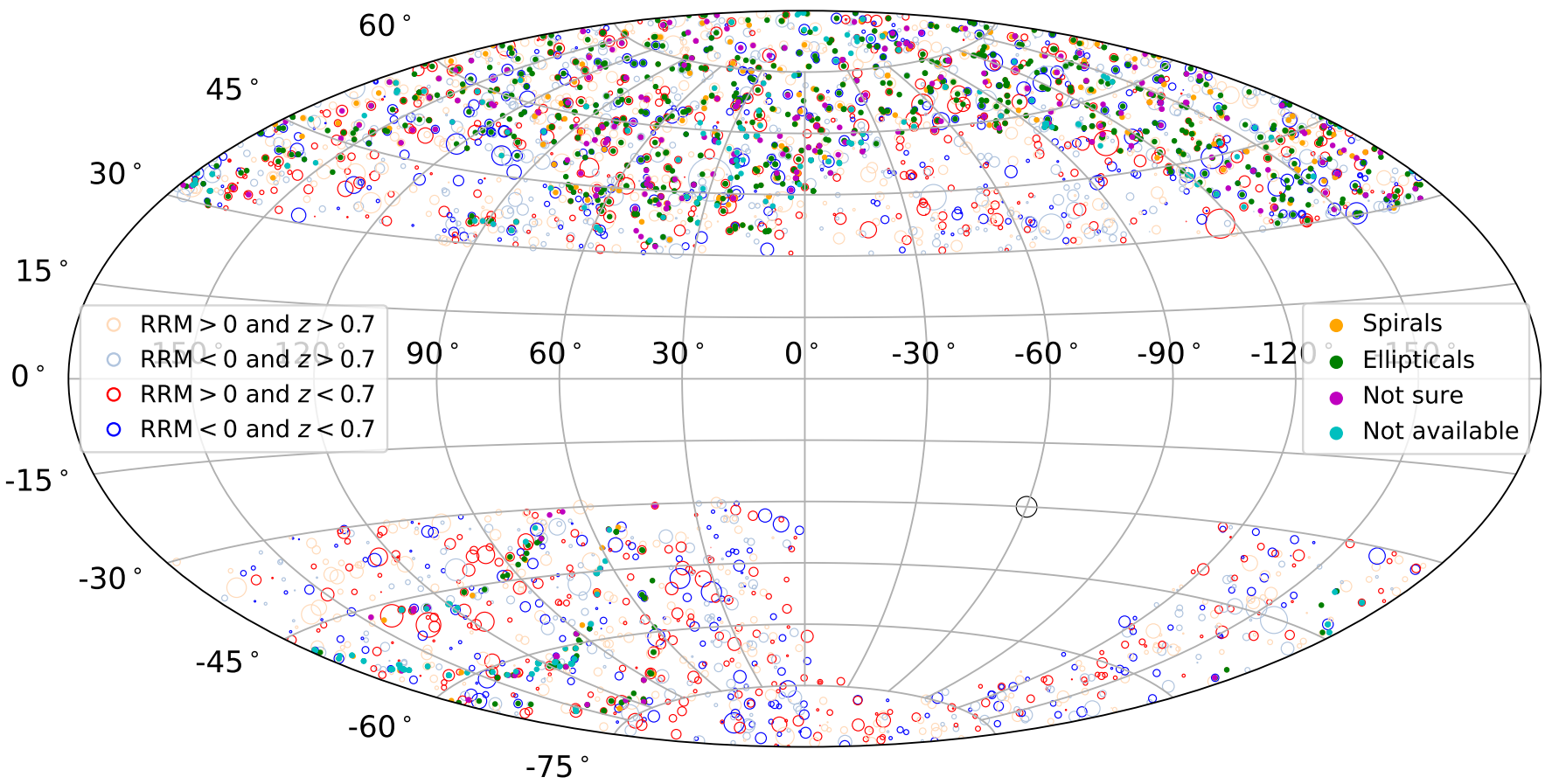

(b) Unfilled circles are the RM sources with positive and negative RRM values with $z<0.7$ or $z>0.7$. The size of the circles is scaled to the magnitude of the RRM of each source. The black circle at coordinates $\left(-60^{\circ},-30^{\circ}\right)$ represents an absolute RRM value of $100 \mathrm{rad} / \mathrm{m}^{2}$. Approximately 3000 background RM sources (available after imposing latitude and redshift availability conditions) are plotted in this figure. Solid dots depict only the intervening galaxies of the four types mentioned in the legend.

Figure 5. All the coordinates in these plots are in the galactic frame. (a) All-sky maps of available galaxies from the SDSS DR16. (b) Available RM sources and known morphology of galaxies in the same region. 


\subsection{Analysis of RRMs via probability distribution functions (PDFs)}

After obtaining the number and morphology of intervening galaxies for RM sources by cross-matching galaxies of known morphology with the RRM sample, we aim to understand the contribution of the $\mathrm{RM}$ from the intervening galaxies to the observed RRM. We would naively expect that on increasing the number of galaxies, the standard deviation of RRM distribution due to background extragalactic polarized sources would increase. Similarly, we would expect that the intervening spiral galaxies would affect the standard deviation more than the elliptical galaxies since the spirals have significantly stronger fields than ellipticals (Sec. 3 and Seta et al. (2021)).

\subsubsection{Gaussian and non-Gaussian RRM PDFs}

The probability density function (PDF) of RM values of the background polarized sources is not completely known and thus is commonly assumed to be a Gaussian distribution, largely described by the mean and standard deviation (see Fig. 1 of Sarala \& Jain (2001), and Fig. 3 and Fig. 7 of Basu et al. (2018) for some Gaussian and non-Gaussian fits and background RM distributions). In this study, we explore the possibility of the PDF to be both a Gaussian and non-Gaussian. Non-Gaussian PDFs, because of the long heavy tail at higher values of $|\mathrm{RM}|$, can better capture the effects of the outliers. Furthermore, the RM distributions from magnetohydrodynamic turbulent simulations and observations can be non-Gaussian (e.g. see Fig. 3a, 6(a), and A1 in Seta \& Federrath 2021). Thus, in addition to computing the statistical parameters of RRM distribution using the data, we fit the RRM probability distribution function with the following three (Gaussian and non-Gaussian distributions with controllable parameters) distributions to obtain statistical parameters of RRM distribution.

- Gaussian distribution $(\mathrm{G})$

$$
\mathrm{g}(\mathrm{RRM})=\frac{1}{(2 \pi)^{1 / 2} \sigma_{\mathrm{RRM}}} \exp \left(\frac{-(\mathrm{RRM}-\mu(\mathrm{RRM}))^{2}}{2 \sigma_{\mathrm{RRM}}^{2}}\right),
$$

where $\mu(\mathrm{RRM})$ and $\sigma_{\mathrm{RRM}}$ represent the mean and standard deviation of the RRM distribution.

- Gaussian distribution with sinh-arcsinh transform (SH)

$$
\operatorname{sh}(y)=\frac{A}{(2 \pi)^{1 / 2} \sigma_{y}} \exp \left(\frac{-\left(y-y_{0}\right)^{2}}{2 \sigma_{y}^{2}}\right)
$$

with

$$
\begin{gathered}
y=\sinh (\delta \operatorname{arcsinh}(\mathrm{RRM})-\epsilon), \text { and } \\
A=\frac{\delta \cosh (\delta \operatorname{arcsinh}(\mathrm{RRM})-\epsilon)}{\left(1+\mathrm{RRM}^{2}\right)^{1 / 2}} .
\end{gathered}
$$

Here, the parameters $\delta$ and $\epsilon$ control the $\mathcal{K}$ (tailedness) and the $\mathcal{S}$ (asymmetry) of the non-Gaussian distribution. When $\delta=1$ and $\epsilon=$ $0, \operatorname{sh}(y)$ turns into a Gaussian distribution. $y_{0}$ and $\sigma_{y}$ are the mean and standard deviation of the distribution, respectively. In a general case, the standard deviation of $\operatorname{sh}(y)$ is not the same as the standard deviation of RRM distribution.

- Modified line-broadening distribution (LB)

$$
\mathrm{lb}(\mathrm{RRM})=\frac{A}{\left(\mathrm{RRM}-u_{0}\right)^{2}+w^{2}} \exp \left(\frac{-\mathrm{RRM}^{2}}{2 \Sigma^{2}}\right)
$$

with

$$
A=\left(\frac{\sqrt{2 \pi} \Sigma}{w^{2}} \cdot \operatorname{voigt}\left(\frac{u_{0}}{w}, \frac{\Sigma^{2}}{2 w^{2}}\right)\right)^{-1},
$$

where voigt function is defined by,

$$
\operatorname{voigt}(x, a)=\frac{1}{\sqrt{4 \pi a}} \cdot \int_{-\infty}^{\infty} \frac{\exp \left(\frac{-(x-y)^{2}}{4 a}\right)}{1+y^{2}} d y .
$$

Here, $w$ and $u_{0}$ are the parameters that control the $\mathcal{K}$ and $\mathcal{S}$ of the distribution, respectively. $\Sigma$ controls the standard deviation of the distribution, and it should be noted that the normalizing parameter $(A)$ is a function of the Voigt function. Also, the parameter $\Sigma$ is not necessarily the same as the standard deviation of the RRM distribution.

In Fig. 6, we show the data and the fits using these functions with one intervening galaxy, $N_{\text {ingal }}=1$, and for general data (data of all galaxies in (a)), spiral data (data of only one intervening spiral galaxy in (b)), and elliptical data (data of only one elliptical galaxy in (c)), respectively, for RM sources with $z<0.7$. We ignore the absolute RRM values greater than $100 \mathrm{rad} / \mathrm{m}^{2}$ to avoid the sensitivity of our results to a few outliers (also see Sec. 2.2.2 in Lan \& Prochaska 2020), just 18 out of 3098 available sources are classified as outliers with this condition (see the top part of Fig. 4). The data is represented with error bars (errors are standard deviations of the variation of mean of every point in a bin calculated by the bootstrapping method with 500 iterations), and the three curves (Eq. 12 for $\mathrm{G}_{\text {fit }}$, Eq. 13a for $\mathrm{SH}_{\text {fit }}$, and Eq. 14a for $\mathrm{LB}_{\text {fit }}$ ) are fitted to the PDFs of the data. Using the fitted probability density functions (G, SH, LB), a sample of $10^{5} \mathrm{RRM}$ points is generated according to its respective probability distribution from the fit for all the three functions. These generated samples can now be utilized to extract various statistical parameters like $\sigma, \mathcal{S}$, and $\mathcal{K}$ (the mean is always very close to zero) of the RRM distribution from the fits. These properties for the data and fits are given in Table 2. The reported kurtosis is such that the kurtosis of a Gaussian distribution is zero. We also perform a $\mathrm{KS}$ test ${ }^{9}$ to compare the RRM distribution obtained from the data with each fitted function. Its test statistic values $\left(D_{\mathrm{KS}}\right)$ and p-values $\left(p_{\mathrm{KS}}\right)$ are given in the last two columns of Table 2, respectively.

The kurtosis of the RRM distribution computed from data is far from zero for all three cases, and thus RRM distribution (and by extension, the RM source distribution) can very well be non-Gaussian. By choosing a confidence level of $95 \%$ (or $p$-value $>0.05$ ), this can also be seen via the last two columns in Table 2, which show via $\mathrm{KS}$ test that the non-Gaussian distributions ( $\mathrm{SH}$ and $\mathrm{LB}$ ) can represent the data equally well as the Gaussian distribution, since the $p_{\mathrm{KS}}>0.05$ for all the distributions. Also, note that the standard deviation of the data is always much larger than the fitted Gaussian because the higher values of RRM are not considered with a Gaussian distribution. So, considering just a Gaussian distribution for rotation measure distribution of background sources is probably incomplete. Thus, we study the dependence of the statistical properties (primarily the standard deviation of the RRM distribution) for all four cases (data, G, SH, and LB) on the number and morphology of intervening galaxies.

It is important to classify the morphology of galaxies which contribute the most. There can be contribution from numerous intervening galaxies, not all of which might contribute significantly. If for a

9 The KS test is performed by calculating the maximum absolute difference between the CDFs of the data and fits. 


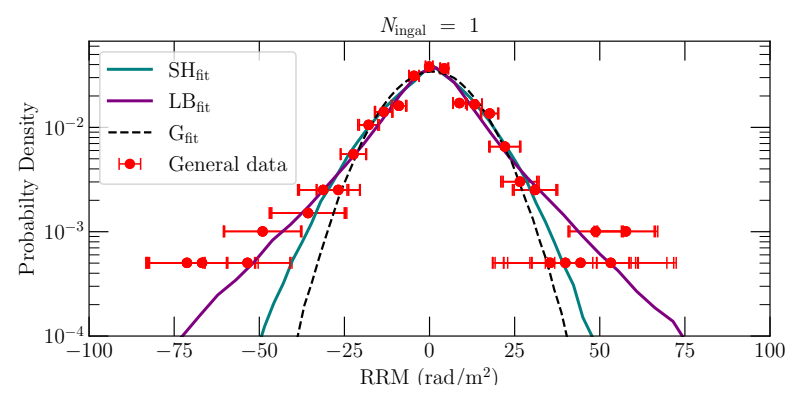

(a) The red curve represents the PDF (with a total of 30 bins) of RRM ( $\mathrm{rad} / \mathrm{m}^{2}$ ) for the background RM sources whose radiation passes through exactly one galaxy inside $\mathrm{R}_{\text {halo/CGM }}$.

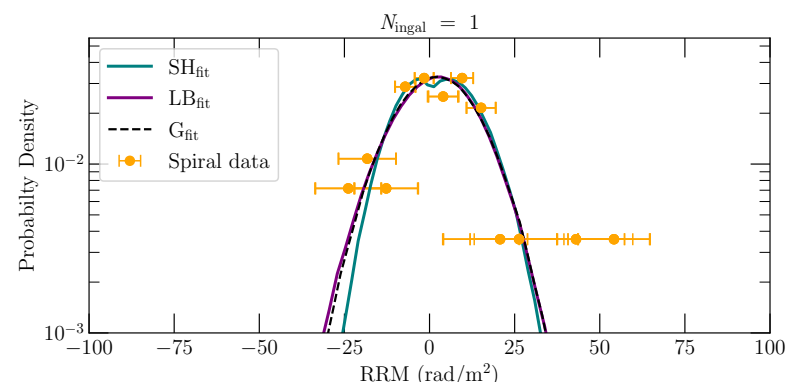

(b) The orange curve represents the PDF (with a total of 15 bins) of $\mathrm{RRM}\left(\mathrm{rad} / \mathrm{m}^{2}\right.$ ) for the background RM sources whose radiation passes through exactly one spiral inside $\mathrm{R}_{\text {halo/CGM }}$. Comparatively shorter tails are observed in this particular PDF, suggesting a lack of outliers.

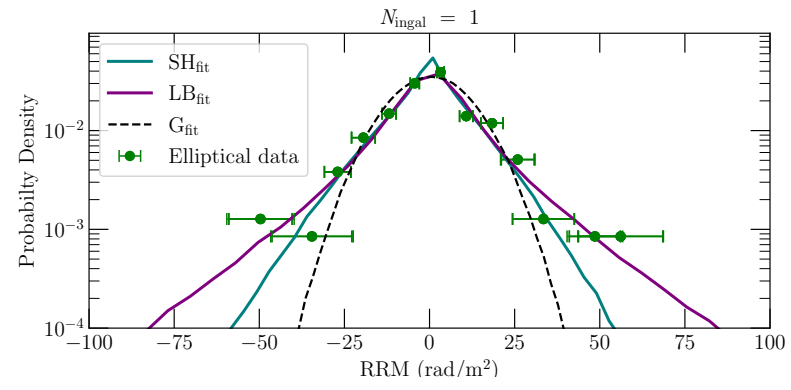

(c) The green curve represents the PDF (with a total of 15 bins) of $\mathrm{RRM}\left(\mathrm{rad} / \mathrm{m}^{2}\right.$ ) for the background RM sources whose radiation passes through exactly one elliptical inside $\mathrm{R}_{\text {halo/CGM }}$.

Figure 6. PDFs of RRM for sources with one intervening galaxy $\left(N_{\text {ingal }}=1\right)$ along the path length and $z<0.7$ for three different cases depending on the morphology: (a) General (all galaxies), (b) spiral, and (c) elliptical. For each case, three different fits using three different fitting functions (Eq. 12 for $\mathrm{G}_{\mathrm{fit}}$, Eq. 13a for $\mathrm{SH}_{\text {fit }}$, and Eq. 14a for $\mathrm{LB}_{\text {fit }}$ ) are created using the dataset of RRM points. All the parameters extracted from the data and fits are provided in Table 2.

particular background source, the number of spirals, $N_{\text {spi }}$, is greater than twice the number of ellipticals, $N_{\text {ell }}$ and twice the number of not sure ones, $N_{\mathrm{NS}}$, the contribution is assumed to be primarily from the spirals. If $N_{\text {ell }} \neq 0$ and $N_{\text {spi }}=N_{\mathrm{NS}}=0$ then the contribution is assumed to be from the ellipticals. If $N_{\mathrm{NS}}>2\left(N_{\mathrm{spi}}+N_{\mathrm{ell}}\right)$ then the contribution is assumed to be from the lenticulars. Only those RM sources with $N_{\mathrm{NA}}=0$ are considered. Here, $N_{\text {spi }}, N_{\text {ell }}, N_{\mathrm{NS}}$, and $N_{\mathrm{NA}}$ refer to the number of intervening 'Spirals', 'Ellipticals', 'Not sure', and 'Not available' galaxies, respectively. All the results containing a morphology classification of the intervening galaxies follow the aforementioned conditions.
Table 2. Statistical properties of the RRM distribution of the data and the fitted functions for each of the three cases in Fig. 6 with one intervening galaxy. The columns are as follows: 1) the morphology of the intervening galaxy: General (all galaxies, Fig. 6(a)), spiral (Fig. 6(b)), and elliptical (Fig. 6(c)), 2) data or the fitted function, 3 ) the computed standard deviation, $\sigma$ in $\left.\mathrm{rad} / \mathrm{m}^{2}, 4\right)$ the computed skewness, $\mathcal{S}, 5$ ) the computed kurtosis, $\mathcal{K}$, such that the kurtosis of a Gaussian distribution is zero, 6) the D-statistic when KS test is performed to compare the RRM data distribution with the fitted function, and 7) the KS test $p$-value.

\begin{tabular}{ccccccc}
\hline Morphology & Case & $\sigma$ & $\mathcal{S}$ & $\mathcal{K}$ & $D_{\mathrm{KS}}$ & $p_{\mathrm{KS}}$ \\
\hline General & Data & 15.21 & -0.22 & 3.49 & - & - \\
General & G & 11.62 & -0.01 & -0.02 & 0.1 & 0.74 \\
General & SH & 13.19 & -0.12 & 0.57 & 0.09 & 0.78 \\
General & LB & 16.33 & -0.07 & 3.82 & 0.08 & 0.82 \\
\hline Spirals & Data & 15.14 & 1.01 & 2.57 & - & \\
Spirals & G & 12.15 & -0.01 & -0.02 & 0.1 & 0.86 \\
Spirals & SH & 11.35 & 0.09 & -0.34 & 0.1 & 0.86 \\
Spirals & LB & 12.36 & -0.08 & 0.1 & 0.1 & 0.86 \\
\hline Ellipticals & Data & 14.57 & 0.13 & 2.36 & - & \\
Ellipticals & G & 11.41 & -0.01 & -0.02 & 0.15 & 0.71 \\
Ellipticals & SH & 13.15 & -0.19 & 2.25 & 0.15 & 0.71 \\
Ellipticals & LB & 17.77 & -0.08 & 5.73 & 0.14 & 0.74 \\
\hline
\end{tabular}

\subsection{Results from RRM PDFs constructed using the background radio sources}

In this section, results obtained from cross-matching the NVSS and SDSS catalogs (Sec. 4.3) are presented ${ }^{10}$.

\subsubsection{Dependence of $\sigma_{\mathrm{RRM}}$ on $N_{\text {ingal }}$}

In this section, we show the dependence of the standard deviation of the RRM distribution, $\sigma_{\text {RRM }}$, on the number of intervening galaxies, $N_{\text {ingal }}$, for three different morphological cases, i.e., general (containing all types of galaxies), spirals, and ellipticals. As described in Sec. 4.4.1, we compute four different $\sigma_{\mathrm{RRM}}$, one directly from the data $\left(\sigma_{\text {data }}\right)$ and other three by fitting the following distributions: Gaussian distribution $\left(\sigma_{\mathrm{G}}\right)$, Gaussian distribution with sinh-arcsinh transform $\left(\sigma_{\mathrm{SH}}\right)$, and modified line-broadening distribution $\left(\sigma_{\mathrm{LB}}\right)$. To calculate one-sigma errors in the statistical parameters, 500 random RRM samples are generated based on one-sigma errors of individual RRM points from the data. The fits are generated for these samples, and the best 250 (least $D_{\mathrm{KS}}$ ) statistical parameters extracted from the fits are considered valid, whose mean and standard deviation are reported as the value and one-sigma fluctuation in the value.

We expect the standard deviation of the RRM distribution $\left(\sigma_{\mathrm{RRM}}\right)$ to increase with the number of intervening galaxies. With the available data, we explore a linear dependence (see Sec. A for a numerical demonstration) of the form

$$
\sigma_{\text {RRM }}\left(N_{\text {ingal }}\right)=A N_{\text {ingal }}+B,
$$

where $A$ is the slope and $B$ is the intercept. Fig. 7 shows the RRM and $\sigma_{\mathrm{RRM}}$ as a function of number of intervening galaxies for all three cases: general (all morphologies included), spirals, and ellipticals. We also provide the slope and intercept of fitted lines for the general cases assuming a linear dependence of $\sigma_{\mathrm{RRM}}$ (for both data and fits) on $N_{\text {ingal }}$ in the captions of Fig. 7a (containing all RM source redshifts) and Fig. 7b (containing RM source redshifts $<0.7$ ). We do not use the $N_{\text {ingal }}=0$ case for fitting the linear slope because many

${ }^{10}$ For all the subsequent figures apart from Fig. 7a, we constrain our analysis to RM sources with $z<0.7$, for robust results (see Sec. 4.3). 


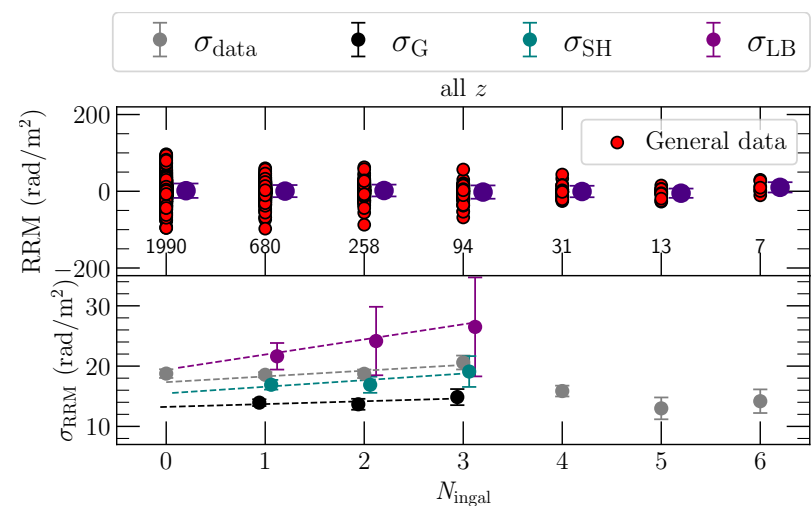

(a) $\sigma_{\text {RRM }}$ vs. $N_{\text {ingal }}$ for the general (all morphologies included, all RM source redshifts) case. The curve-fitting functions are used for just $N_{\text {ingal }}=1-3$, due to lack of datapoints at higher $N_{\text {ingal }}$. The slope values for data, $\mathrm{G}, \mathrm{SH}$, and $\mathrm{LB}$ cases are $0.97 \pm 1.23,0.41 \pm 1.5,1.1 \pm$ 2.66 , and $2.65 \pm 8.61$ respectively, and their corresponding intercepts are $17.32 \pm 1.97,13.25 \pm 2.51,15.45 \pm 4.1$, and $18.93 \pm 13.12$.

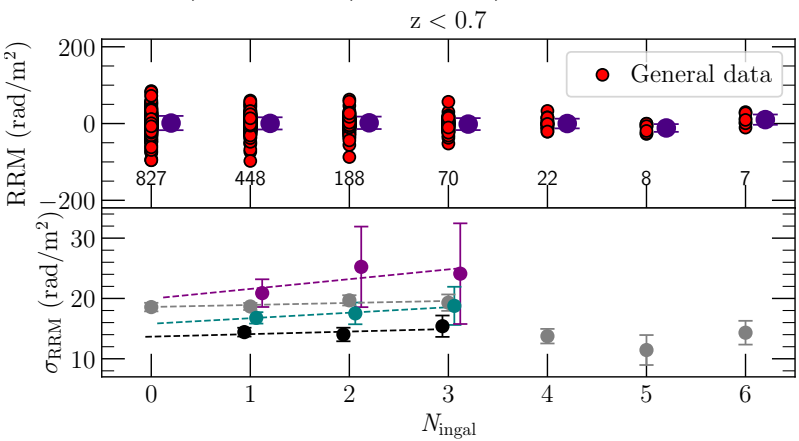

(b) $\sigma_{\text {RRM vs. }} N_{\text {ingal }}$ for the general (with RM source $z<0.7$ ) case. The slope values for data, G, SH, LB cases are $0.31 \pm 1.48,0.57 \pm$ $1.98,1.05 \pm 3.3$, and $1.24 \pm 8.34$, and their corresponding intercepts are $18.57 \pm 2.49,13.56 \pm 3.26,15.57 \pm 5.14$, and $20.9 \pm 13.02$.

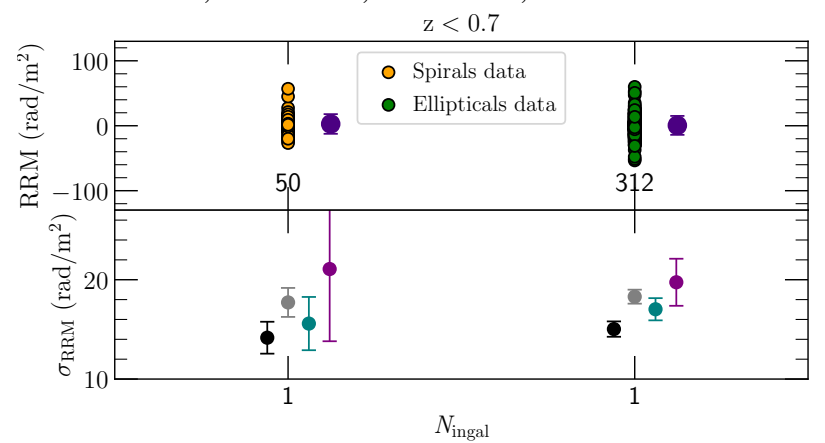

(c) $\sigma_{\text {RRM }}$ vs. $N_{\text {ingal }}$ for just intervening spirals and ellipticals (with RM source $z<0.7, N_{\text {ingal }}=1$ ) case.

Figure 7. The dependence of RRM (top panels in each subfigure) and $\sigma_{\text {RRM }}$ (bottom panels in each subfigure) on $N_{\text {ingal }}$ for three different intervening galaxy samples based on morphology: all galaxies, (a) and (b) with all RM source $z$ and $z<0.7$, respectively, and spirals and ellipticals, (c). All the top panels show RRM scatter with the violet point showing $\mu_{\mathrm{RRM}}$ and its errorbar showing $\sigma_{\mathrm{RRM}}$ for each case. The number of sources in each sample is also given in the plot. The bottom panels show $\sigma_{\text {RRM }}$ as a function of $N_{\text {ingal }}$ for data and three different fitting distributions (described in Sec. 4.4.1). The offsets in $N_{\text {ingal }}$ for different cases of $\sigma$ are introduced to improve the legibility of the plot. The error in $\sigma_{\mathrm{RRM}}$ is computed using the RM uncertainty from the NVSS (Farnes et al. 2014a). For most cases, $\sigma_{\text {RRM }}$ increases with an increase in the number of intervening galaxies. However, the range of $N_{\text {ingal }}$ is quite small (upto 6 for the general case). of those sources might not have any reported intervening galaxies due to the lack of coverage in SDSS-DR16 data (see Fig. 5).

In Fig. 7a and Fig. 7b, we do not overlap our data with results from fitting the distribution beyond $N_{\text {ingal }}=3$, as the number of RRM values are quite low and the fitting procedure does not give statistically significant results. The slope tends to be positive (correlation of $\sigma_{\text {RRM }}$ with $N_{\text {ingal }}$ is consistent with results using MgII absorbers in Farnes et al. (2014b); Malik et al. (2020)) for the data and all three distributions of the general case (Fig. 7a, Fig. 7b), but it is difficult to conclude that robustly because of large errors in the estimated values. This is probably due to a smaller number of galaxies for each $N_{\text {ingal }}$, fewer number of $N_{\text {ingal }}$ values ( 3 for the general case), and large errors in the observed RRM values.

The linear dependence of $\sigma_{\text {RRM }}$ with $N_{\text {ingal }}$ is different from what the study of Lan \& Prochaska (2020) assumed, i.e., $\sigma_{\mathrm{RRM}} \propto \sqrt{N_{\text {ingal }}}$. The difference arises because they assume a random walk model of RRM even at a smaller number of intervening galaxies, which we think is probably applicable only at a very high number of intervening galaxies. They also find a higher number of intervening galaxies $(\sim$ 10 , they do not differentiate between the morphology of intervening galaxies) from their sample (see Fig. 2 in their paper). However, we find only up to six intervening galaxies for our general case. Moreover, they do not observe any correlation of $\sigma_{\text {RRM }}$ with $N_{\text {ingal }}$. Amaral et al. (2021) performs a similar analysis with no significant correlation detected as well. According to our fitting functions (LB function in particular), there can be a positive correlation between the two (see the slope in the captions of Fig. 7a, Fig. 7b), however, due to large error in slope values it might also be uncorrelated. Thus, we need further data to conclude this concretely. The differences in the results might also be because of the different galaxy surveys (we use SDDS DR 16, and they use DESI Legacy Imaging Surveys) ${ }^{11}$.

We also classify the SDSS catalog based on galaxy's morphology using the Galaxy Zoo data as we expect significant differences between thermal electron density and magnetic fields in spiral and elliptical galaxies. In Fig. 7c, we show the same analysis for just intervening spirals and ellipticals $\left(N_{\text {ingal }}=1\right)$, with no notable differences seen between the $\sigma_{\mathrm{RRM}}$ of the two. The number of ellipticals (312) is much higher than the number of spirals (50) because the hosts of the RM sources (mainly ellipticals) are also included as intervening galaxies.

Even though magnetic fields cannot be accurately derived using this section's data due to large errors, and lack of data points, we consider a Gaussian and two non-Gaussian distributions for the RRM PDFs. From non-Gaussian PDFs, we observe that the outliers can play a role in determining the $\sigma_{\mathrm{RRM}}$. This can be seen in Fig. 7 as $\sigma_{\mathrm{LB}}>\sigma_{\mathrm{SH}}>\sigma_{\mathrm{G}}$ for nearly all the cases. Also, from Table 2 LB function tends to have higher kurtosis and lower $D_{\mathrm{KS}}$ values, meaning it fits the outliers well. We believe that this is the reason for higher values of $\sigma_{\mathrm{LB}}$. However, more data with higher sensitivity is required to confirm these claims.

\subsubsection{Dependence of $\sigma_{\mathrm{RRM}}$ on the impact parameter $\left(\mathrm{r}_{\perp}\right)$}

Here, we explore the dependence of $\sigma_{\mathrm{RRM}}$ on the impact parameter, $r_{\perp}$ (distance from the center of the galaxy to a region where the line of sight from the sources crosses the galaxy). We assume $\mathrm{R}_{\text {halo/CGM }}$ as the extent to which a galaxy can contribute, which is fixed to a

11 A possible cause of differences could be the redshift range, which is $0<z<0.7$ for the SDSS dataset (spectroscopic), and $0.3<z<1.0$ for the Legacy survey (photometric). 


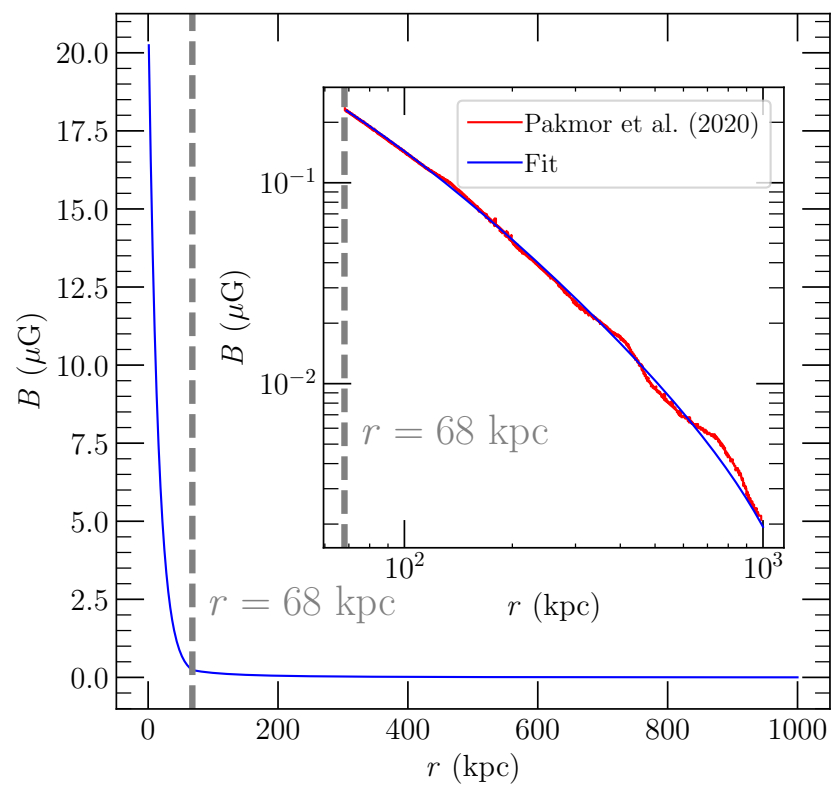

Figure 8. The adopted large-scale field $(B)$ profile for spirals. Below $r=68 \mathrm{kpc}$ an exponentially decaying field is taken. Above $r=$ $68 \mathrm{kpc}$, profile from Pakmor et al. (2020) (for circumgalactic medium) is obtained and fitted, as shown in the inset. The fitted function $0.592 \exp \left(-\log \left(3.03\left(r(\mathrm{kpc}) / r_{\mathrm{vir}}\right)+0.617\right) / 0.54\right)-0.00163$ is used as the profile above $r=68 \mathrm{kpc}$.

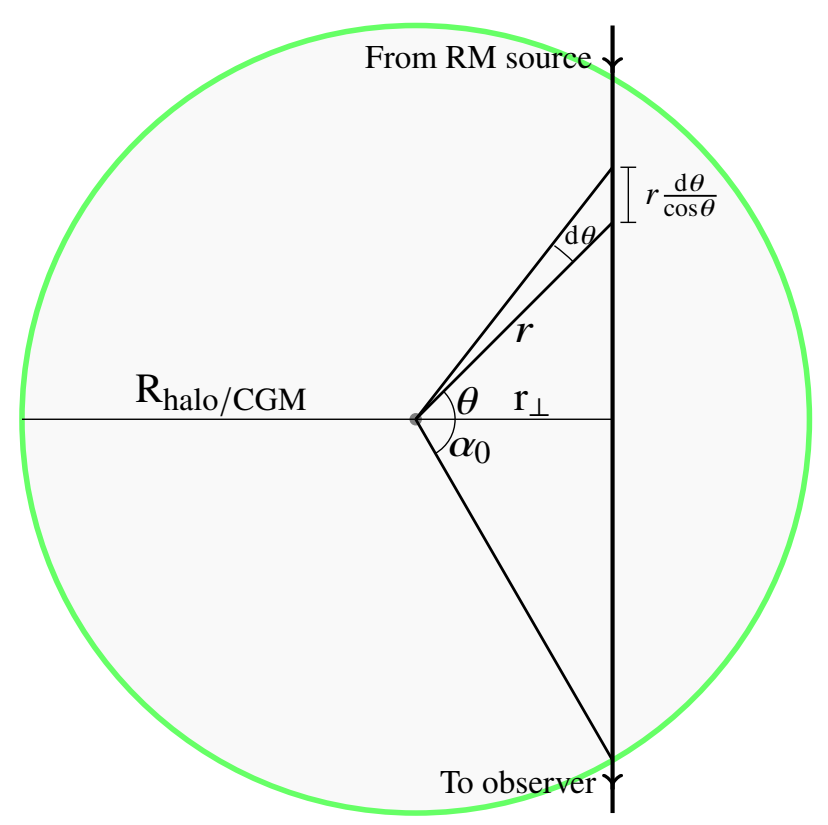

Figure 9. The green circle represents the bounds of an intervening galaxy. In the figure, $R_{\text {halo/CGM }}$ and $r_{\perp}$ represent the radius of the galaxy's halo (10 $\mathrm{R}_{\mathrm{gal}}$ ) and the impact parameter, respectively. The radial distance $r$ changes as the radiation traverses through the medium, and $2 \alpha_{0}$ is the angle subtended by the path length (vertical line) at the center. The differential element along the path length is $1 / \cos \theta$ times the differential arc at $r$, i.e., $r \mathrm{~d} \theta$. maximum of $1 \mathrm{Mpc}$ for both ellipticals and spirals. $\mathrm{R}_{\text {halo/CGM }}$ is taken as ten times the Petrosian radius $\left(R_{\mathrm{gal}}\right)$, which varies with the galaxy. In our sample, the minimum $\mathrm{R}_{\text {halo/CGM }}$ for spirals and ellipticals is approximately $46 \mathrm{kpc}$ and $31 \mathrm{kpc}$, respectively. The mean and standard deviations of $\mathrm{R}_{\text {halo/CGM }}$ are $580 \mathrm{kpc}$ and $360 \mathrm{kpc}$ for spirals, and $447 \mathrm{kpc}$ and $334 \mathrm{kpc}$ for ellipticals. The study of Lan \& Prochaska (2020) contains a similar analysis but for a fixed $\mathrm{R}_{\text {halo/CGM }}(200 \mathrm{kpc}$ ) for all galaxies (see their Fig. 4), whereas we vary the upper limit of the impact parameter depending on the size of the individual galaxy. Thus, we normalize $r_{\perp}$ by the Petrosian radius, $\mathrm{R}_{\text {gal }}$ (ip ratio $)$.

We also use simple analytical radial $(r)$ profiles of the thermal electron density and magnetic fields in spirals to estimate the standard deviation of the RRM distribution as a function of the impact parameter. For spiral galaxies, we adopt the following thermal electron density profile for the disk (Taylor \& Cordes 1993) and the halo or CGM ( $r$ in kpc)

$$
\begin{aligned}
n_{\mathrm{e}}(r) & =0.0223 \mathrm{~cm}^{-3}\left(\operatorname{sech}\left(\frac{r}{20 \mathrm{kpc}}\right)\right)^{2} \\
& +0.1 \exp \left(\left(-\frac{r-3.5 \mathrm{kpc}}{1.8 \mathrm{kpc}}\right)^{2}\right), r \leq 68 \mathrm{kpc}, \\
n_{\mathrm{e}}(r) & =0.01 \mathrm{~cm}^{-3}\left(1+\left(\frac{r}{3 \mathrm{kpc}}\right)^{2}\right)^{-3 / 4}, r>68 \mathrm{kpc},
\end{aligned}
$$

respectively. According to the profile from Taylor \& Cordes (1993), the disk $n_{\mathrm{e}}$ component falls to $10^{-4} \mathrm{~cm}^{-3}$ at $r=68 \mathrm{kpc}$ and thus we assume a king profile for the halo/CGM after that limit (since the features of spirals become more homogeneous). The constant $0.01 \mathrm{~cm}^{-3}$ in Eq. 17 is obtained by demanding continuity in the overall $n_{\mathrm{e}}$ profile around $r=68 \mathrm{kpc}$. For large-scale magnetic field strengths in spiral galaxies, we use an exponentially decaying profile (Basu et al. $2018)$, i.e. $B_{0}=21.6 \mu \mathrm{G} \exp (-r / 15 \mathrm{kpc})$ up to $r=68 \mathrm{kpc}$ with field reversals at every $15 \mathrm{kpc}$. Beyond $68 \mathrm{kpc}$, we adopt a fit to the profile of CGM from Pakmor et al. (2020) in simulated Milky Way type spiral galaxies (see the top panel of their Fig. 13) ${ }^{12}$. The constant $21.6 \mu \mathrm{G}$ is obtained by demanding continuity in the overall $B$ profile, and is consistent with the observationally derived magnetic field strengths in Fig. 3 of Basu \& Roy (2013). Fig. 8 shows the two profiles adopted for the core and halo/CGM of spirals separated by the grey line. The fitting function (see inset of Fig. 8) obtained is $0.592 \exp \left(-\log \left(3.03\left(r(\mathrm{kpc}) / r_{\text {vir }}\right)+0.617\right) / 0.54\right)-0.00163$, where $r_{\mathrm{vir}}=200 \mathrm{kpc}$ is the virial radius of the galaxy (Pakmor et al. 2020). This analysis is not performed for ellipticals as their large-scale field profiles are not very well-known, and we obtain extremely low magnitudes for large-scale field strengths from the Laing-Garrington analysis (see Fig. 3).

Using these models in Eq. 2 and the geometry in Fig. 9, we compute the RM contribution of the intervening galaxy as

$\frac{\mathrm{RM}_{\text {ingal }}}{\mathrm{rad} / \mathrm{m}^{2}}=\frac{811.9}{(1+z)^{2}} \int_{-\alpha_{0}}^{\alpha_{0}} \frac{n_{e}\left(\mathrm{r}_{\perp} / \cos \theta\right)}{\mathrm{cm}^{-3}} \frac{B\left(\mathrm{r}_{\perp} / \cos \theta\right) / \sqrt{3}}{\mu \mathrm{G}} \frac{2 \mathrm{r}_{\perp} / \pi}{\mathrm{kpc}} \frac{\mathrm{d} \theta}{\cos ^{2} \theta}$,

In this equation, we divide the large-scale field magnitudes by $\sqrt{3}$ to determine the component parallel to the line of sight, assuming an isotropic field in all three directions. The impact parameter is multiplied with a factor of $2 / \pi$ to account for the galaxies' random

12 We use the WebPlotDigitizer, available at https://automeris.io/ WebPlotDigitizer/, to extract data from Pakmor et al. (2020). 

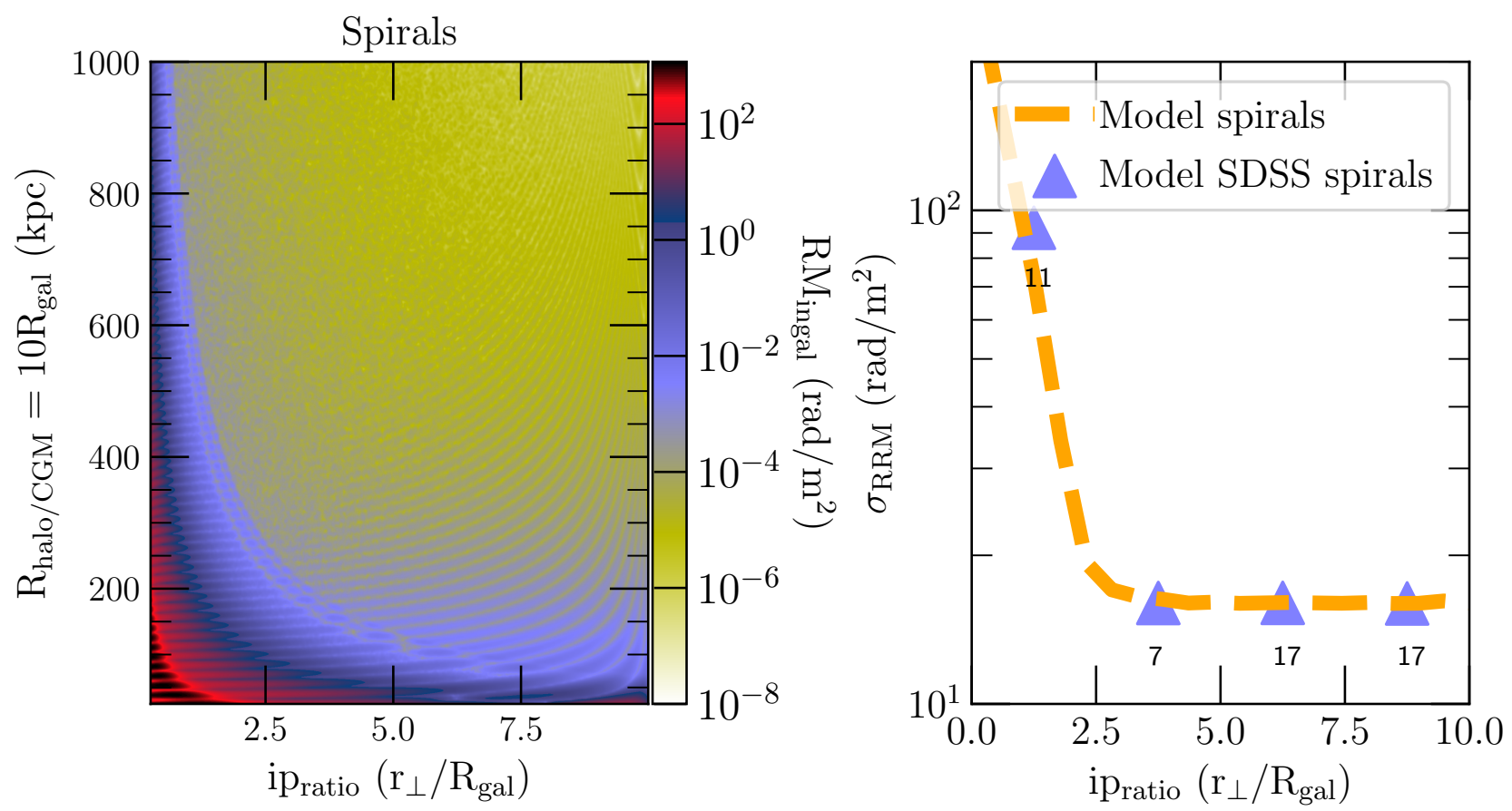

Figure 10. Left: The histogram represents predictions of $\left|\mathrm{RM}_{\text {ingal }}\right|$ with the model with varying $\mathrm{R}_{\text {halo/CGM }}$ and ip ${ }_{\text {ratio }}$, thermal densities, and large-scale fields.

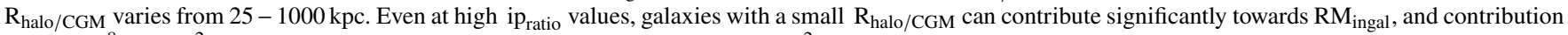
below $10^{-8} \mathrm{rad} / \mathrm{m}^{2}$ is considered as zero. The highest $\mathrm{RM}_{\text {ingal }}$ is $\approx 1000 \mathrm{rad} / \mathrm{m}^{2}$, occurring near the origin of the figure. Right: The variation of $\sigma_{\mathrm{RRM}}$ with $\mathrm{ip}_{\text {ratio }}$, assuming a Gaussian background source distribution of $\mathrm{RM}$ with a standard deviation of $16 \mathrm{rad} / \mathrm{m}^{2}$ for all galaxies with $\mathrm{R}_{\text {halo }} / \mathrm{CGM}$ from $25 \mathrm{kpc}$ to $1000 \mathrm{kpc}$, is shown with the orange curve. Then, ip ratio varying from $0-10$ is divided into four equally sized bins. The dependence of $\sigma_{\text {RRM }}$ on ip ratio for SDSS spirals is shown with blue triangles, which varies similarly as the model spirals in each bin. However, after removing one galaxy with $\mathrm{RM}$ ingal $=300 \mathrm{rad} / \mathrm{m}^{2}$, the $\sigma_{\text {RRM }}$ of model SDSS spirals varies only slightly. This explains the uncertainty in the positive slope correlation in Fig. $7 \mathrm{~b}$.

alignment with the line of sight (Basu et al. 2018). The integration limits $-\alpha_{0}$ to $\alpha_{0}$ correspond to the entire path length as can be seen in Fig. 9. $z$ is taken as 1 to generate the model in Fig. 10. The maximum computed value for the spirals goes up to $\approx 1000 \mathrm{rad} / \mathrm{m}^{2}$ with a very low probability of occurrence $(\sim 0.001)$, which is consistent with the predictions of Basu et al. (2018) (see the left panel of their Fig. 7). The left panel of Fig. 10 shows the variation of this modeled $\mathrm{RM}_{\text {ingal }}$ with $\mathrm{ip}_{\text {ratio }}$ and $\mathrm{R}_{\text {halo/CGM}}$.

We assume a Gaussian distribution of rotation measures from the background sources with a standard deviation of $16 \mathrm{rad} / \mathrm{m}^{2}$ (selected as this is the standard deviation of RRM at very large impact parameters of the order of $500 \mathrm{kpc}$ ) and randomly impart our modeled $\mathrm{RM}_{\text {ingal }}$ (using Eq. 18) along varying ip ratio $_{\text {by considering }}$ the presence of all the $\mathrm{R}_{\text {halo/CGM }}$ from our model sample in the range $25 \mathrm{kpc}$ to $1000 \mathrm{kpc}$ (according to the left panel of Fig. 10). These are called model spirals. Then we select the spiral galaxies from the SDSS dataset and predict their $\mathrm{RM}_{\text {ingal }}$ from the model (using Eq. 18) with the help of their extracted sizes and impact parameters from the observational dataset. These are called model SDSS spirals. The right panel of Fig. 10 shows the predictions for both of these. The match between the two is significant. However, one galaxy with $\mathrm{RM}_{\text {ingal }}=300 \mathrm{rad} / \mathrm{m}^{2}$ causes the rise in $\sigma_{\mathrm{RRM}}$ in the first bin of the modeled SDSS spirals case. After removing that galaxy, the maximum $\sigma_{\text {RRM }}$ of the modeled SDSS spirals is $17.2 \mathrm{rad} / \mathrm{m}^{2}$, which is too sensitive to be observed (errors in Fig. 7 are greater than $\sim 2.5 \mathrm{rad} / \mathrm{m}^{2}$ ).

Additionally, the scarcity of SDSS spirals in the former bins explains the low values of predicted $\sigma_{\mathrm{RRM}}$ (without considering the galaxy with $\left.\mathrm{RM}_{\text {ingal }}=300 \mathrm{rad} / \mathrm{m}^{2}\right)^{13}$. As most of the galaxies are situated in the later bins (where $\mathrm{RM}_{\text {ingal }}$ is low), the overall contribution of SDSS spirals in $\sigma_{\mathrm{RRM}}$ is not observable. This explains the large uncertainties in the positive slope correlation (see Fig. 7b) observed in Sec. 4.5.1. The ambiguity can be resolved with deeper observations with a sufficient number of galaxies present in each ip ratio $_{\text {bin. }}$

\subsubsection{Influence of intervening galaxies on the polarization fraction}

In addition to rotation measure values, we also use the available observed polarization fractions in the Farnes et al. (2014a) dataset. We use the morphologically classified galaxy catalog to explore the influence of intervening spirals and ellipticals on the polarization fractions of the background sources. Fig. 11 shows a scatter plot of the distribution of polarization fraction values of radiation traversing through spirals and ellipticals with different $N_{\text {ingal }}$, and the mean of the distributions are shown above the points. The mean polarization fraction values for spirals are lower than that of the ellipticals, implying stronger magnetic fields and higher thermal electron density in spirals (making them strong depolarizers). We exploit the difference in the values of polarization fractions between spirals and ellipticals to estimate the ratio of magnetic field strengths between the two samples.

The thermal electron density profile for spirals is modeled by

\footnotetext{
13 Former bins with lower $\mathrm{ip}_{\text {ratio }}$ can contribute the highest to $\sigma_{\mathrm{RRM}}$.
} 


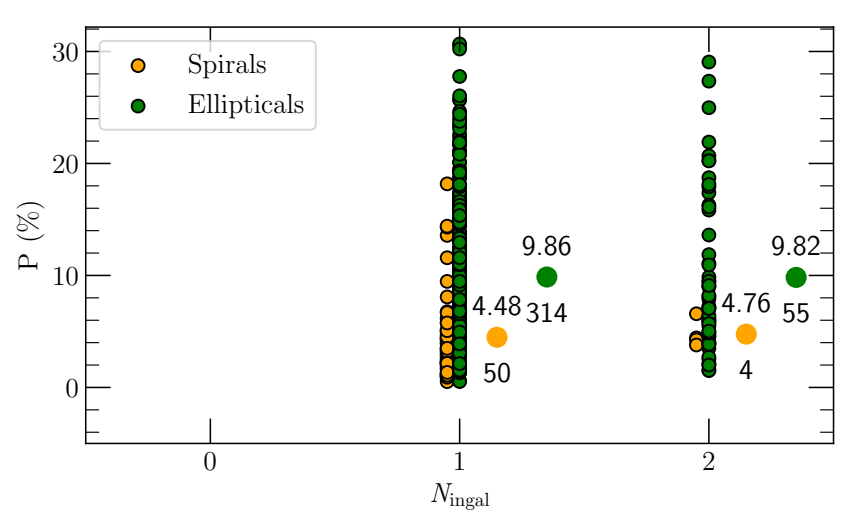

Figure 11. Polarization fraction, $\mathrm{P}(\%)$, as a function of the number of intervening galaxies, $N_{\text {ingal }}$, for spirals (orange) and ellipticals (green). For each case, the mean is plotted with dots and their magnitude above it. The numbers below that point show the sample size in each case. Ellipticals tend to have a high polarization fraction than spirals. This implies, statistically, spirals have stronger magnetic fields and higher thermal electron densities.

Eq. 16 and Eq. 17 (Taylor \& Cordes 1993), and for elliptical galaxies, we use the King profile

$$
n_{\mathrm{e}}(r)=0.1 \mathrm{~cm}^{-3}\left(1+\left(\frac{r}{3 \mathrm{kpc}}\right)^{2}\right)^{-3 / 4},
$$

where the constant $0.1 \mathrm{~cm}^{-3}$ is consistent with Mathews \& Brighenti (2003). For spirals, we adopt the flux-freezing condition with $b_{\text {rms }} \propto n_{\mathrm{e}}^{2 / 3}$ and limit the maximum $b_{\text {rms }}$ to $10 \mu \mathrm{G}$ to calculate the proportionality constant. Then we use these models to estimate the $\sigma_{\mathrm{RM}}$ using single-scale model of magnetic field structures (Burn 1966; Felten 1996; Laing et al. 2008b). The $\sigma_{\mathrm{RM}}^{2}$ (according to the geometry in Fig. 9) is

$$
\sigma_{\mathrm{RM}}^{2}=\frac{811.9^{2} \ell_{b} C^{2}}{3(1+z)^{2}} \int_{-\alpha_{0}}^{\alpha_{0}} n_{\mathrm{e}}^{2(1+2 / 3)}\left(\mathrm{r}_{\perp} / \cos \theta\right) \mathrm{r}_{\perp} \frac{\mathrm{d} \theta}{\cos ^{2} \theta},
$$

where $\mathrm{r}_{\perp}$ and $\ell_{b}$ are in $\mathrm{kpc}, \ell_{b}$ is the magnetic field correlation length, and $C$ is the proportionality constant in the equation $b_{\text {rms }} \propto$ $n_{\mathrm{e}}^{2 / 3}$ profiles ( $C_{\mathrm{spi}}$ for spirals and $C_{\mathrm{ell}}$ for ellipticals). All the other units are the standard Gaussian units as used throughout the paper. We represent Eq. 20 as $\sigma_{\mathrm{RM}}^{2}=T C^{2}$ to separate the proportionality constant and other galaxy dependent terms ( $T_{\text {spi }}$ for spirals and $T_{\text {ell }}$ for ellipticals). Since $\sigma_{\mathrm{RM}}^{2}$ is an additive quantity (Felten 1996; Laing et al. 2008a), we half the value predicted by Eq. 20 for the identified hosts $^{14}$ as, on average, the polarized emission emerges halfway in the plane of observation in the sample of host galaxies.

Then we take an average of logarithms of polarization values (see Eq. 3) passing through spirals and ellipticals and subtract the two to remove any contribution from the IGM and source (which are statistically similar for both samples). The only unknown in the subtracted equations is the proportionality constant $\left(C_{\mathrm{ell}}\right)$ of $b_{\mathrm{rms}}$ of the ellipticals. The other parameters are known from the NVSSSDSS cross-matching, correlation lengths $\left(\ell_{b}=30\right.$ pc for spirals, $\ell_{b}=100 \mathrm{pc}$ for ellipticals), and the assumed $b_{\mathrm{rms}}$ profile of the spirals. From this analysis, $C_{\mathrm{ell}}$ is

14 The intervening galaxies in our study also contain the host galaxies. To identify them, we use the condition $\Delta z=\left|z_{\mathrm{RM}}-z_{\text {gal }}\right|<0.001$. If multiple hosts are identified for one RM source, the galaxy with the lowest $\Delta z$ is identified as the host.

$$
C_{\text {ell }}^{2}=\frac{\left\langle C_{\text {spi }}^{2} \sum_{k=1}^{N_{\text {ingal }}} T_{\text {spi }_{i k}}\right\rangle+\frac{\left\langle\log \left(P_{\text {spi }_{i}}\right)\right\rangle-\left\langle\log \left(P_{\text {ell }}\right)\right\rangle}{2 \lambda^{4}}}{\left\langle\sum_{k=1}^{N_{\text {ingal }}} T_{\text {ell }_{j}}\right\rangle},
$$

where $i$ and $j$ denote the background sources with radiation passing through spirals and ellipticals, respectively. $k$ sums over 1 or 2 intervening galaxies (see Fig. 11) for a particular source $i$ or $j$. This equation automatically accounts for galaxies with high ip ratio which contribute less as their calculated $\sigma_{\mathrm{RM}}^{2}$ contribution will be low (according to Eq. 20).

We extract $b_{\text {rms }}$ profile for ellipticals from the calculated $C_{\text {ell }}$ $(\sim 29.51)$ as $b_{\text {rmsell }}=C_{\mathrm{ell}} n_{\mathrm{e}}^{2 / 3}$, which turns out to be $b_{\text {rmsell }}(r)=$ $6.36 \mu \mathrm{G}\left(1+(r / 3)^{2}\right)^{-1 / 2}$ after assuming a King profile for their $n_{\mathrm{e}}$ (see Eq. 19). The extracted $b_{\text {rms }}(0)$ for ellipticals is approximately $6.36 \mu \mathrm{G}$ which is in agreement with the results of previous studies like Mathews \& Brighenti (2003) and Seta et al. (2021).

\section{CONCLUSIONS}

We aim to estimate magnetic fields in elliptical galaxies from observations, primarily in their haloes and circumgalactic medium (CGM), using the Laing-Garrington effect and polarized emission from background radio sources.

For the Laing-Garrington effect (Sec. 3), we find that the estimated small-scale magnetic field strengths in the sample of galaxies with redshifts ranging from 0.01 to 0.46 lie in the range $0.06-2.75 \mu \mathrm{G}$. The magnetic field strengths in ellipticals are an order of magnitude smaller than that in the Milky Way and nearby spiral galaxies. We find that the large-scale field, albeit over larger path lengths of the order of $100 \mathrm{kpc}$, is an order of magnitude smaller than the random magnetic fields (Fig. 3). A larger sample of Laing-Garrington sources with a higher signal-to-noise ratio of the polarized intensity is required to study these magnetic fields in much greater detail.

With observations of polarized background radio sources, we aim to explore the effects of morphology and number of intervening galaxies on the residual rotation measure, RRM (the observed RM after subtracting the modeled Milky Way contribution). Most previous studies assume that the RM distribution of background sources is Gaussian, and so we first explore the possibility of non-Gaussian distributions. We show that non-Gaussian RM distributions might be better (Fig. 6) as they can account for large rotation measure values. We propose that the standard deviation of the RRM distribution has a linear dependence on the number of intervening galaxies, $N_{\text {ingal }}$, for a lower number of intervening galaxies but goes as $\sigma_{\text {RRM }} \propto \sqrt{N_{\text {ingal }}}$ for a very high $N_{\text {ingal }}$ (according to results from Sec. A). Then we find that $\sigma_{\mathrm{RRM}}$ values (computed directly from the data, fitting a Gaussian distribution, and two different non-Gaussian distributions) tend to increase with $N_{\text {ingal }}$ (aligned with the results from $\mathrm{MgII}$ absorber studies in Bernet et al. (2008); Farnes et al. (2014b); Malik et al. (2020)). But since the errors in the estimated slopes (Fig. 7) are high, $\sigma_{\text {RRM }}$ can as well be independent of $N_{\text {ingal }}$ (as seen in Lan \& Prochaska (2020); Amaral et al. (2021)). We then use simple radial profiles of magnetic fields and thermal electron density for spirals in the data to show that the contribution of the rotation measure of the intervening galaxy to $\sigma_{\mathrm{RRM}}$ is negligible. This is probably because the impact parameter is very large for most of the spirals in our sample. However, we do use the difference in the observed polarization fractions between intervening spirals and ellipticals to obtain a field strength of $\sim 6.3 \mu \mathrm{G}$ at the center of ellipticals (which is consistent with the previous studies (Mathews \& Brighenti 2003; Seta et al. 2021)). A much larger sample with lesser uncertainty in the rotation 
measure observations is required to study the probability distribution function of RRM and compute higher-order statistical properties of the distribution. This would robustly confirm the presence of nonGaussianity, which in turn would also help us study magnetic fields in galaxies in more detail.

Both the methods have their pros and cons. They both probe the small-scale magnetic fields via their depolarization signatures; however, the Laing-Garrington method cannot individually (for every source) distinguish whether the radiation is depolarized due to the galactic medium or the ICM/IGM surrounding the galaxies. On the contrary, radiation from the background polarized sources passing through intervening galaxy is individually constrained to be inside the galactic halo/CGM scales. Although, the Laing-Garrington method works without assuming any small-scale magnetic field profile. It is, however, necessary to assume the flux freezing condition $\left(b_{\mathrm{rms}} \propto n_{\mathrm{e}}^{\gamma}\right.$ ) to derive results from the observations of polarized sources.

Both methods utilize very different datasets and analysis techniques but give similar answers for the strength of magnetic fields in ellipticals, which is of the order of $1-10 \mu \mathrm{G}$. The results can be used to further constrain fluctuation dynamo theories (Rincon 2019) and also parameters of the magnetohydrodynamic simulations of galaxy formation and evolution (such as Pakmor et al. 2020; Nelson et al. 2020).

\section{ACKNOWLEDGEMENTS}

We thank both anonymous reviewers for their helpful feedback. We also thank Sui Ann Mao and Aritra Basu for valuable discussions. We acknowledge the use of high-performance computing resources provided by the Research School of Astronomy and Astrophysics at the Mount Stromlo Observatory.

\section{DATA AVAILABILITY}

Radio galaxies' data used for the Laing-Garrington analysis (Sec. 3.1) has been derived from Vernstrom et al. (2019). The data is available at https://iopscience.iop.org/0004-637X/878/2/92/ suppdata/apjab1f83t1_mrt.txt. For the background polarized emission analysis, there are three datasets: RM catalog (Sec. 4.1), galaxy catalog (Sec. 4.2), and morphology classification of galaxies' catalog (Galaxy Zoo). The RM catalog is derived from Farnes et al. (2014a) (NVSS data), galaxy catalog from Ahumada et al. (2020) (SDSS DR16 data), and galaxy zoo data from Lintott et al. (2008). To remove the Milky Way RM contribution $\left(\mathrm{RM}_{\mathrm{MW}}\right)$, we adopted Oppermann's maps (Oppermann et al. 2012, data available at https: //wwwmpa .mpa-garching.mpg.de/ift/ faraday/2014/index.html) and Faraday 2020 (data available at https://wwwmpa.mpa-garching.mpg.de/ ensslin/ research/data/faraday2020.html). The SDSS catalogs along with the Galaxy Zoo data were found and queried on the Catalog Archive Server (Thakar et al. 2008) at https: //skyserver . sdss . org/casjobs/. The analyzed data is available upon a reasonable request to the author, Amit Seta (amit.seta@anu.adu.au).

\section{REFERENCES}

Ahn C. P., et al., 2012, ApJS, 203, 21

Ahumada R., et al., 2020, ApJS, 249, 3

Amaral A. D., Vernstrom T., Gaensler B. M., 2021, MNRAS,
Banfield J. K., et al., 2015, Monthly Notices of the Royal Astronomical Society, 453, 2326

Basu A., Roy S., 2013, MNRAS, 433, 1675

Basu A., Mao S. A., Fletcher A., Kanekar N., Shukurov A., Schnitzeler D., Vacca V., Junklewitz H., 2018, MNRAS, 477, 2528

Beck R., 2007, A\&A, 470, 539

Beck R., 2015, A\&ARv, 24, 4

Beck R., Brandenburg A., Moss D., Shukurov A., Sokoloff D., 1996, ARA\&A, 34,155

Becker R. H., White R. L., Helfand D. J., 1995, ApJ, 450, 559

Berkhuijsen E. M., Müller P., 2008, A\&A, 490, 179

Bernet M. L., Miniati F., Lilly S. J., Kronberg P. P., Dessauges-Zavadsky M., 2008, Nature, 454, 302

Bhat P., Subramanian K., 2013, MNRAS, 429, 2469

Birnboim Y., Balberg S., Teyssier R., 2015, MNRAS, 447, 3678

Blackman E. G., 1998, ApJ, 496, L17

Brandenburg A., Subramanian K., 2005, Phys. Rep., 417, 1

Burn B. J., 1966, MNRAS, 133, 67

Cesarsky C. J., 1980, ARA\&A, 18, 289

Condon J. J., Cotton W. D., Greisen E. W., Yin Q. F., Perley R. A., Taylor G. B., Broderick J. J., 1998, AJ, 115, 1693

Crocker A. F., Bureau M., Young L. M., Combes F., 2011, MNRAS, 410, 1197

Dabhade P., Combes F., Salomé P., Bagchi J., Mahato M., 2020, A\&A, 643, A111

Dawson K. S., et al., 2016, AJ, 151, 44

Duric N., 1988, Space Sci. Rev., 48, 73

Evirgen C. C., Gent F. A., Shukurov A., Fletcher A., Bushby P., 2017, MNRAS, 464, L105

Farnes J. S., Gaensler B. M., Carretti E., 2014a, ApJS, 212, 15

Farnes J. S., O'Sullivan S. P., Corrigan M. E., Gaensler B. M., 2014b, ApJ, 795,63

Federrath C., 2016, Journal of Plasma Physics, 82, 535820601

Federrath C., Chabrier G., Schober J., Banerjee R., Klessen R. S., Schleicher D. R. G., 2011, Phys. Rev. Lett., 107, 114504

Federrath C., Schober J., Bovino S., Schleicher D. R. G., 2014, ApJ, 797, L19

Felten J. E., 1996, in Trimble V., Reisenegger A., eds, Astronomical Society of the Pacific Conference Series Vol. 88, Clusters, Lensing, and the Future of the Universe. p. 271

Fletcher A., 2010, in Kothes R., Landecker T. L., Willis A. G., eds, Vol. 438, The Dynamic Interstellar Medium: A Celebration of the Canadian Galactic Plane Survey. p. 197 (arXiv: 1104.2427)

Fletcher A., Beck R., Shukurov A., Berkhuijsen E. M., Horellou C., 2011, MNRAS, 412, 2396

Gaensler B. M., Haverkorn M., Staveley-Smith L., Dickey J. M., McClureGriffiths N. M., Dickel J. R., Wolleben M., 2005, Science, 307, 1610

Gaensler B. M., Madsen G. J., Chatterjee S., Mao S. A., 2008, Publ. Astron. Soc. Australia, 25, 184

Garrington S. T., Conway R. G., 1991, MNRAS, 250, 198

Garrington S. T., Leahy J. P., Conway R. G., Laing R. A., 1988, Nature, 331, 147

Gerhard O., 2010, Galaxies and their Masks, p. 339-346

Grasso D., Rubinstein H. R., 2001, Phys. Rep., 348, 163

Hamilton T. S., Turnshek D. A., Casertano S., 2000, in American Astronomical Society Meeting Abstracts. p. 109.02

Hammond A. M., Robishaw T., Gaensler B. M., 2012, arXiv e-prints, p. arXiv: 1209.1438

Herzenberg A., 1958, Philosophical Transactions of the Royal Society of London Series A, 250, 543

Ho L. C., 2009, ApJ, 699, 626

Hopkins P. F., et al., 2018, MNRAS, 480, 800

Hutschenreuter S., et al., 2021, arXiv e-prints, p. arXiv:2102.01709

Jiang Y.-F., Ciotti L., Ostriker J. P., Spitkovsky A., 2010, The Astrophysical Journal, 711, 125

Kazantsev A. P., 1968, Soviet Journal of Experimental and Theoretical Physics, 26, 1031

Kierdorf M., et al., 2020, A\&A, 642, A118

Klein U., Fletcher A., 2015, Galactic and Intergalactic Magnetic Fields 
Krumholz M. R., Federrath C., 2019, Frontiers in Astronomy and Space Sciences, 6, 7

Krumholz M. R., Burkhart B., Forbes J. C., Crocker R. M., 2018, MNRAS, 477, 2716

Laing R. A., 1988, Nature, 331, 149

Laing R. A., Bridle A. H., Parma P., Murgia M., 2008a, MNRAS, 391, 521

Laing R. A., Bridle A. H., Parma P., Murgia M., 2008b, MNRAS, 391, 521

Lan T.-W., Prochaska J. X., 2020, MNRAS, 496, 3142

Larmor J., 1919, Reports of the British Association, 87, 159

Li M., Li Y., Bryan G. L., Ostriker E. C., Quataert E., 2020a, ApJ, 894, 44

Li M., Li Y., Bryan G. L., Ostriker E. C., Quataert E., 2020b, ApJ, 898, 23

Lilly S. J., Prestage R. M., 1987, MNRAS, 225, 531

Lintott C. J., et al., 2008, MNRAS, 389, 1179

Lintott C., et al., 2011, MNRAS, 410, 166

Ma Y. K., Mao S. A., Stil J., Basu A., West J., Heiles C., Hill A. S., Betti S. K., 2019, MNRAS, 487, 3454

Mac Low M.-M., Klessen R. S., 2004, Reviews of Modern Physics, 76, 125

Majewski S. R., et al., 2017, AJ, 154, 94

Malarecki J. M., Staveley-Smith L., Saripalli L., Subrahmanyan R., Jones D. H., Duffy A. R., Rioja M., 2013, MNRAS, 432, 200

Malik S., Chand H., Seshadri T. R., 2020, ApJ, 890, 132

Mathews W. G., Brighenti F., 1997, The Astrophysical Journal, 488, 595

Mathews W. G., Brighenti F., 2003, ARA\&A, 41, 191

Matthaeus W. H., Qin G., Bieber J. W., Zank G. P., 2003, ApJ, 590, L53

Moss D., Shukurov A., 1996, MNRAS, 279, 229

Nelson D., et al., 2020, MNRAS, 498, 2391

Nyland K., et al., 2017, MNRAS, 464, 1029

Oppermann N., et al., 2012, A\&A, 542, A93

Oppermann N., et al., 2015, A\&A, 575, A118

Owen F. N., Laing R. A., 1989, MNRAS, 238, 357

Pakmor R., et al., 2020, MNRAS, 498, 3125

Putman M. E., Peek J. E. G., Joung M. R., 2012, ARA\&A, 50, 491

Raymond J. C., 1992, ApJ, 384, 502

Rincon F., 2019, Journal of Plasma Physics, 85, 205850401

Ruzmaikin A. A., Sokoloff D. D., Shukurov A. M., eds, 1988, Magnetic fields of galaxies Astrophysics and Space Science Library Vol. 133, doi:10.1007/978-94-009-2835-0.

Sarala S., Jain P., 2001, MNRAS, 328, 623

Sarazin C. L., White Raymond E. I., 1988, ApJ, 331, 102

Saripalli L., 2012, The Astronomical Journal, 144, 85

Schekochihin A. A., Cowley S. C., Taylor S. F., Maron J. L., McWilliams J. C., 2004, ApJ, 612, 276

Seta A., 2019, PhD thesis, Newcastle University, Newcastle Upon Tyne, UK, http://theses.ncl.ac.uk/jspui/handle/10443/4685

Seta A., Federrath C., 2020, MNRAS, 499, 2076

Seta A., Federrath C., 2021, MNRAS, 502, 2220

Seta A., Shukurov A., Wood T. S., Bushby P. J., Snodin A. P., 2018, MNRAS, 473,4544

Seta A., Bushby P. J., Shukurov A., Wood T. S., 2020, Physical Review Fluids, 5,043702

Seta A., Rodrigues L. F. S., Federrath C., Hales C. A., 2021, ApJ, 907, 2

Shukurov A., Sokoloff D., 2007, in Cardin P., Cugliandolo L. F., eds, Vol. 88, Les Houches, Session LXXXVIII, Dynamos. Amsterdam: Elsevier, pp 251-299, doi:10.1016/S0924-8099(08)80008-X, http://dx. doi. org/10.1016/S0924-8099(08) 80008-X

Shukurov A., Snodin A. P., Seta A., Bushby P. J., Wood T. S., 2017, ApJ, 839, L16

Sokoloff D. D., Bykov A. A., Shukurov A., Berkhuijsen E. M., Beck R., Poezd A. D., 1998, MNRAS, 299, 189

Tabatabaei F. S., Krause M., Fletcher A., Beck R., 2008, A\&A, 490, 1005

Taylor J. H., Cordes J. M., 1993, ApJ, 411, 674

Taylor A. R., Stil J. M., Sunstrum C., 2009, ApJ, 702, 1230

Thakar A. R., Szalay A., Fekete G., Gray J., 2008, Computing in Science and Engineering, 10, 30

Tumlinson J., Peeples M. S., Werk J. K., 2017, ARA\&A, 55, 389

Uson J. M., Boughn S. P., Kuhn J. R., 1990, Science, 250, 539

Vernstrom T., Gaensler B. M., Rudnick L., Andernach H., 2019, ApJ, 878, 92

\begin{tabular}{ccccc}
\hline Distributions & $\mu$ & $\sigma_{\text {RRM }}$ & $\mathcal{S}$ & $\mathcal{K}$ \\
\hline G & -0.07 & 20.04 & -0.01 & -0.02 \\
SH & -0.09 & 19.98 & -0.04 & 2.71 \\
LB & -0.09 & 20.05 & -0.05 & 3.17 \\
\hline
\end{tabular}

Table A1. Parameters of the initial background RM distributions. We have fixed the $\sigma_{\text {RRM }}$ to be $20 \mathrm{rad} / \mathrm{m}^{2}$, and the $\mu$ and $\mathcal{S}$ to be 0 . The slight variations from the fixed values in second decimal places are a result of the finite number of elements in a sample.

Wright E. L., et al., 2010, AJ, 140, 1868

Yao J. M., Manchester R. N., Wang N., 2017, ApJ, 835, 29

de Gouveia Dal Pino E. M., 2006, in Herrera-Velázquez J. J. E., ed., American Institute of Physics Conference Series Vol. 875, Plasma and Fusion Science: 16th IAEA Technical Meeting on Research using Small Fusion Devices. pp 289-295 (arXiv:astro-ph/0603065), doi:10.1063/1.2405951

van de Voort F., Bieri R., Pakmor R., Gómez F. A., Grand R. J. J., Marinacci F., 2021, MNRAS, 501, 4888

\section{APPENDIX A: BACKGROUND RM METHOD SIMULATION}

In this section, we explore the dependence of $\sigma_{\text {RRM }}$ on $N_{\text {ingal }}$ via simple numerical simulations. We create an ideal dataset of 50000 background sources with their RM distribution following the three (Gaussian and non-Gaussian) probability density functions $(\mathrm{G}, \mathrm{SH}$, and LB) discussed in Sec. 4.4 (check Fig. 6 for their application on the SDSS data). The parameters of these distributions are set such that the standard deviation of the rotation measure is approximately $20 \mathrm{rad} / \mathrm{m}^{2}$ for all three of them. This is motivated by the fact that the intercepts of slopes when applied to the SDSS data (see Fig. 7a and Fig. $7 \mathrm{~b}$ ) lie in the range $15-24 \mathrm{rad} / \mathrm{m}^{2}$. Table A1 contains the statistical properties, such as the mean $\mu$, standard deviation $\sigma$, skewness $\mathcal{S}$, and kurtosis $\mathcal{K}$, of the background RM distribution for all three cases.

To simulate the contribution of intervening galaxies, a random $\mathrm{RM}$ of the intervening galaxy, $\mathrm{RM}_{\text {ingal }}$, in the range $4-11 \mathrm{rad} / \mathrm{m}^{2}$ (either positive or negative) is assigned randomly to every intervening galaxy. The probability density functions (PDFs) of RRM for 50000 sources for $N_{\text {ingal }}$ ranging from 1 to 10 , considering all three different background distributions, are shown in Fig. Ala. The dispersion $\left(\sigma_{\mathrm{RRM}}\right)$ increases as the no. of intervening galaxies $\left(N_{\text {ingal }}\right)$ increases (as the curve spreads more at higher $N_{\text {ingal }}$ ).

We assume the dependence of $\sigma_{\mathrm{RRM}}$ on $N_{\text {ingal }}$ of the form

$$
\sigma_{\text {RRM }}\left(N_{\text {ingal }}\right)=\mathrm{A}\left(N_{\text {ingal }}\right)^{\mathrm{n}}+\mathrm{B},
$$

where $\mathrm{A}, \mathrm{B}, \mathrm{n}$ are to be determined. We fit the simulated data for upto $500 N_{\text {ingal }}$ with $\mathrm{RM}_{\text {ingal }}$ randomly selected in the range $4-11 \mathrm{rad} / \mathrm{m}^{2}$ (sign also selected randomly). The fitting parameters $(\mathrm{A}, \mathrm{B}, \mathrm{n})$ of Eq. A1 do not vary with the background source distribution (decided by $\mathrm{G}, \mathrm{SH}$, and LB functions), as evident from Fig. A1b as $\mathrm{n}=0.61$ for all the fits. Eq. A1 is an excellent fit as seen from the overlapping curves of Fig. A1b for $\mathrm{n}=0.61$, this is close to the expectation that $\sigma_{\text {RRM }} \propto \sqrt{N_{\text {ingal }}}($ Lan \& Prochaska 2020). We emphasize this is only true for a very large number of background sources and intervening galaxies.

As Eq. A1 does not vary with the background source distributions, we take a Gaussian background of RM sources and calculate the fitting parameters from Eq. A1 for different $\mathrm{RM}_{\text {ingal }}$ ranges as shown in Fig. A1c. In a realistic scenario $N_{\text {ingal }}=500$ is a rarity, hence we also compute Eq. A1 for different $N_{\text {ingal }}$. There is an increasing trend 


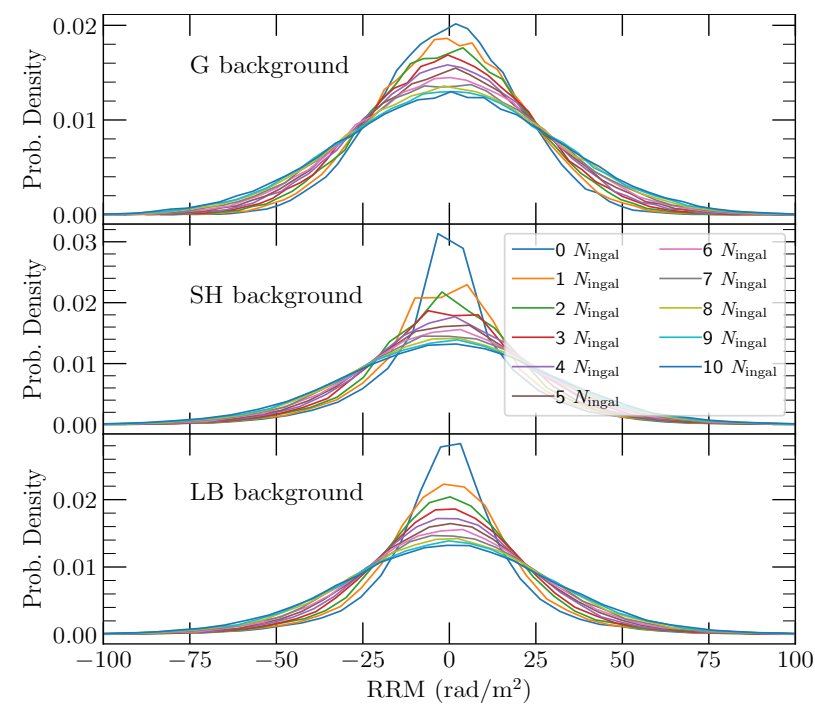

(a) The PDF distributions with $|\mathrm{RRM}|<100 \mathrm{rad} / \mathrm{m}^{2}$ having either $\mathrm{G}, \mathrm{SH}, \mathrm{LB}$ distributions as initial background with random $\mathrm{RM}_{\text {ingal }}$ contributions between $4-11 \mathrm{rad} / \mathrm{m}^{2}$ attributed to every intervening galaxy for a background source.

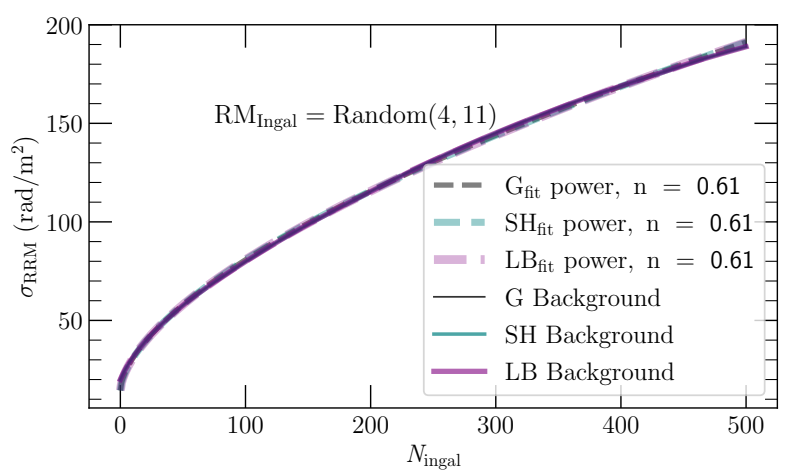

(b) For $\mathrm{RM}_{\text {ingal }}$ between 4 and $11 \mathrm{rad} / \mathrm{m}^{2}, \sigma_{\mathrm{RRM}} \mathrm{VS} N_{\text {ingal }}$ reveals that the properties of the curves are independent of the background source distribution, as all the six curves (the simulated data, and its curve-fits according to Eq. A1) perfectly overlap over each other.

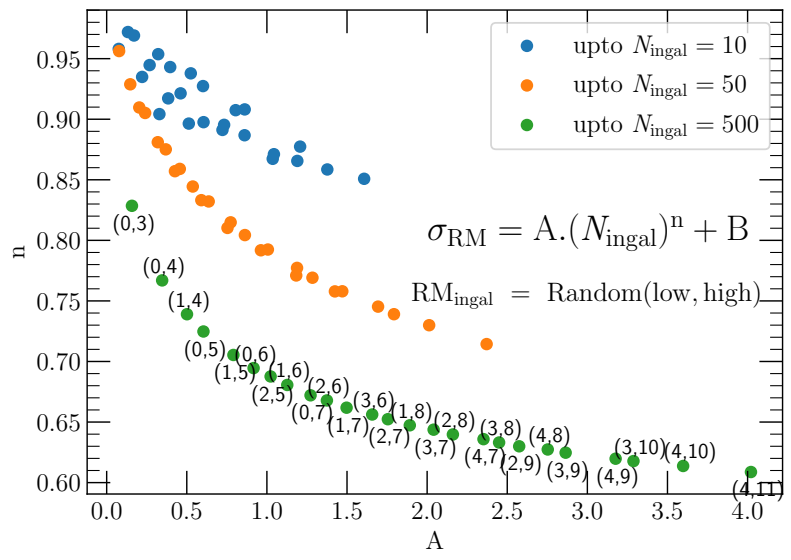

(c) Variation of A and $\mathrm{n}$ from Eq. A1 with varying $\mathrm{RM}_{\text {ingal }}$. Random(low, high) selects random $\mathrm{RM}_{\text {ingal }}$ between low and high and annotated brackets represent these values. Due to congestion, the brackets are not annotated below the blue and orange dots, but they occur in the same order as the green dots. For low $N_{\text {ingal }}$ fit, the power $(\mathrm{n}) \approx 0.9$, implying an almost linear dependence. of power (n) when $N_{\text {ingal }}$ reduces. For $N_{\text {ingal }}$ up to 10, the power (n) is $\approx 0.9$, implying that Eq. A1 is almost a linear equation. From the actual SDSS data, we only receive data upto $N_{\text {ingal }}=6$ (see Fig. 7a), thus $\sigma_{\text {RRM }}$ dependence on $N_{\text {ingal }}$ can be safely assumed as linear for low $N_{\text {ingal }}$.

This paper has been typeset from a $\mathrm{T}_{\mathrm{E}} \mathrm{X} / \mathrm{LT} \mathrm{E} \mathrm{X}$ file prepared by the author. 